\title{
Transcriptomic profiling and genetic analyses reveal novel key regulators of cellulase and xylanase gene expression in Penicillium oxalicum
}

\author{
Yu-Si Yan ${ }^{\dagger}$, Shuai Zhao ${ }^{* \dagger}$, Lu-Sheng Liao ${ }^{\dagger}$, Qi-Peng He ${ }^{\dagger}$, Ya-Ru Xiong, Long Wang, Cheng-Xi Li \\ and Jia-Xun Feng ${ }^{*}$ (1)
}

\begin{abstract}
Background: The transition to a more environmentally friendly economy has prompted studies of modern biorefineries, including the utilization of low-value lignocellulose. The major challenge facing the widespread application of biorefineries is the high cost of enzymes that can efficiently hydrolyze recalcitrant cellulose to sugars. Penicillium oxalicum produces large amounts of plant-cell-wall-degrading enzymes, but their production is tightly controlled by complex regulatory networks, resulting in low yields of the native enzymes. Regulatory genes have been the targets of genetic engineering to improve enzyme production in microorganisms. In this study, we used transcriptomic profiling and genetic analyses to screen for and identify novel key regulators of cellulase and xylanase gene expression in P. oxalicum.

Results: A comparative analysis of the transcriptomes of $P$. oxalicum HP7-1 on different carbon sources, including glucose, wheat bran, and wheat bran plus Avicel, identified 40 candidate genes regulating the expression of cellulolytic enzyme genes. Deletion mutants of 31 candidate genes were constructed in P. oxalicum $\triangle P$ PoxKu70 and 11 resultant mutants showed significant changes in their filter-paper cellulase production compared with the parental strain $\triangle P O X K u 70$. Among these 11 mutants, $\triangle P O X C X r A, \triangle P O X C \times r B$, and $\triangle P O X N s d D$ showed the most significant reduction in the enzyme production $(96.8,75.9$, and $58.5 \%$, respectively). Ten of these 11 genes are here reported to be involved in cellulase production for the first time. Further tests revealed that $\triangle P$ OxC $\times$ rA,$\triangle P o x C \times r B$, and $\triangle P O x N s d D$ displayed significantly reduced xylanase production, whereas $\triangle P O x C x r A$ produced negligible xylanase. Interestingly, $\triangle P O X C x r B$ and $\triangle P O X N s d D$ showed significantly increased $\beta$-glucosidase production. Real-time quantitative reverse transcription-PCR and an electrophoretic mobility shift assay (EMSA) showed that PoxCXrA, PoxCXrB, and PoxNsdD regulate the expression of one another, but the mode of regulation changes dynamically during the growth of fungal cells in the presence of cellulose. EMSA showed that PoxCxrA, PoxCxrB, and PoxNsdD directly bind the putative promoters of major cellulase and xylanase genes.
\end{abstract}

Conclusions: We have detected and identified three key new regulatory genes, PoxCxrA, PoxCxrB, and PoxNsdD, that directly and indirectly regulate the expression of cellulase and xylanase genes in $P$. oxalicum. This study provides novel insights into the regulatory mechanisms of fungal cellulase and xylanase gene expression.

Keywords: Penicillium oxalicum, Transcriptomic profiling, Transcription factor, Cellulase, Xylanase, Regulation

\footnotetext{
*Correspondence: shuaizhao0227@gxu.edu.cn; jiaxunfeng@sohu.com

${ }^{\dagger}$ Yu-Si Yan, Shuai Zhao, Lu-Sheng Liao and Qi-Peng He contributed equally to this work

State Key Laboratory for Conservation and Utilization of Subtropical Agro-bioresources, College of Life Science and Technology, Guangxi University, 100 Daxue Road, Nanning 530004, Guangxi, People's Republic of China
} 


\section{Background}

Plant cell walls, which consist primarily of cellulose, hemicellulose, and lignin, are the most abundant renewable bioresource on earth. Filamentous fungi, particularly Penicillium, Trichoderma, Aspergillus, and Neurospora species, produce a diverse array of enzymes in response to different ecological niches. These include plant-cellwall-degrading enzymes (CWDEs) that depolymerize the main structural polysaccharide components of plant cell walls into single sugars (glucose or xylose), which can be further converted into liquid fuels and/or other useful chemicals. However, low CWDE production has seriously retarded the industrialization of lignocellulosic biorefineries [1].

Penicillium has already received serious attention as an alternative to Trichoderma reesei for the production of industrial cellulases for use in lignocellulose saccharification. It has two major advantages over $T$. reesei: high $\beta$-glucosidase (BGL) activity in extracellular cellulase systems and its superior hydrolytic performance, which is dependent on the high specific activity of cellobiohydrolase 1 (CBH1) [2]. In the genus Penicillium, P. oxalicum HP7-1, isolated from a decayed subtropical forest soil system in China, produces high cellulase activity for alkaline-pretreated sugarcane bagasse [3, 4], which is the major waste product in the Chinese sugar industry. The whole genomic sequence of $P$. oxalicum HP7-1 and its annotation were recently completed, and showed that it has an integrative lignocellulolytic enzyme system for the degradation of plant cell walls, with diverse components [5].

The complexity of the signaling cascades involved and our incomplete knowledge of the relevant transcriptional regulatory networks have hindered the improvement of native CWDE production in filamentous fungi [6]. The CWDE genes are coordinately but differentially regulated in their hosts. The further characterization and manipulation of regulatory networks of CWDE gene expression should allow CWDE yields to be improved via the rational genetic engineering of filamentous fungi.

The transcriptional regulation of cellulolytic gene expression is vital for cellulase biosynthesis and secretion in filamentous fungi, and depends on a regulatory network of multiple positively and negatively acting transcription factors (TFs). This network controls cellulolytic and xylanolytic gene expression in response to multiple external inducers/repressors, such as low-molecularweight monosaccharides or disaccharides produced by the degradation of polysaccharides, light signaling, and $\mathrm{pH}$ [7].

$\mathrm{X} \ln \mathrm{R}$ is the first-identified TF involved in xylan and cellulose degradation, and regulates the expression of xylanolytic and cellulolytic genes induced by D-xylose or cellobiose in Aspergillus [8, 9]. Several TFs have subsequently been identified as involved in the degradation of plant biomass. These predominantly include transcription activators, such as CLR-1/ClrA, CLR-2/ClrB [5, 10, 11], VIB1/XprG [12, 13], and Ace3 [14], and carbon catabolite repressors, such as CreA/CRE1/CRE-1 [1517]. The TF Ace 3 is a master regulator of cellulase gene expression and a modulator of xylanase gene expression, which is mediated partly by the XlnR homologue Xyr1 in T. reesei [14]. In N. crassa, CLR-2 functions as a primary transcriptional activator, directly regulating the transcription of cellulolytic enzyme genes. The transcriptional regulation of $c l r-2$ is controlled by activated CLR-1, and CLR-1 and CLR-2 together constitute the full responses to cellulase gene expression $[10,15]$. Another TF, VIB1, indirectly activates the CLR-1/CLR2-triggered expression of cellulolytic enzyme genes by repressing both Cre1-mediated carbon catabolite repression (CCR) and COL-26/BglR-mediated glucose sensing and metabolism in the early phase of cellulolytic induction [12]. CCR is a global regulatory mechanism that ensures microorganisms, including filamentous fungi, preferentially utilize glucose or other rapidly metabolizable carbon sources over less-favorable carbohydrates. In filamentous fungi that can degrade lignocellulose, such as Aspergillus, Trichoderma, Penicillium, and Neurospora, the C2H2-type TF CreA/CRE1/CRE-1 functions as the main repressor in CCR. It directly or indirectly represses the expression of almost all CWDE genes and their TFs involved in the degradation of plant biomass in the presence of glucose [2, 15-17]. In A. nidulans, the CreA function depends in part on de novo protein synthesis, and CreA expression is not only autoregulated, but is also regulated by $\mathrm{CreB}-\mathrm{CreC}$-mediated ubiquitination [18]. COL-26 plays an important role in regulating glucose metabolism in $N$. crassa, and functions synergistically with CRE-1 to regulate cellulase gene expression [12].

TFs and their homologues in different fungi also play diverse roles in the regulation of CWDE gene expression, conferring specificity and diversity on the mechanisms regulating the expression of these genes. For example, $\mathrm{Xyr} 1 / \mathrm{X} \ln R$ activates the transcription of the cellulase and xylanase genes in T. reesei, $A$. niger, and $P$. oxalicum, whereas $\mathrm{Xlr}-1 / \mathrm{X} \ln \mathrm{R}$ only regulates the expression of xylanase genes in N. crassa and A. nidulans [15]. In $N$. crassa, CLR-1 promotes the transcription of a variety of genes encoding cellulases, hemicellulases, cellodextrin transporters, and proteins involved in protein secretion, whereas its homologue ClrA is less involved in the regulation of cellulase and hemicellulase gene expression in $A$. nidulans $[7,10]$.

In $P$. oxalicum, many TFs regulating the expression of cellulase and hemicellulase genes have been identified, 
including POX02484, POX08522, Ace1, AmyR, Bgl2, CreA, ClrB, ClrB-2, ClrC, and XlnR [5, 17]. Of these, $\mathrm{ClrB}$, CreA, XlnR, and AmyR are important dosedependent TFs in cellulase production. During the induction of cellulose or cellodextrins, CreA and AmyR repress the expression of cellulase genes, whereas $\mathrm{ClrB}$ and $\mathrm{X} \ln \mathrm{R}$ activate their expression. In the early stage of induction, $\mathrm{ClrB}$ represses $a m y R$ expression, which is activated by CreA. AmyR also plays a negative role in the regulation of $c \operatorname{lr} B$ and $x \ln R$ expression. The transcriptional expression of $a m y R, \operatorname{clr} B$, and $x \ln R$ is also repressed by CreA in the presence of glucose [17].

The combined manipulation of TFs and their target genes recently enhanced the production of cellulolytic enzymes in P. oxalicum. The simultaneous deletion of the TF-encoding genes $c r e A$ and $b g l 2$ and the overexpression of $\operatorname{clr} B$ improved the filter-paper cellulase (FPase) activity and its extracellular protein concentration over 20- and 10 -fold, respectively [19]. When the gene encoding the $\mathrm{TF} \mathrm{X} \ln \mathrm{R}^{\mathrm{A} 871 \mathrm{~V}}, \operatorname{clr} B$, and two major cellulase genes, $c b h 1$ and $e g 1$, were overexpressed and the carbon catabolite repressor gene $c r e A$ was deleted, the engineered $P$. oxalicum strain showed approximately tenfold higher cellulase activity than the wild type [20]. These data suggest that genetically modifying TFs by overexpressing or deleting their genes can efficiently improve the production of cellulolytic enzymes. However, these improvements cannot meet the industrial demand for lignocellulosic biorefineries. Therefore, more-specific regulators of cellulase and xylanase gene expression in P. oxalicum are still required. These should also will extend our understanding of the mechanisms regulating cellulase and xylanase gene expression and how $P$. oxalicum can be engineered to enhance its cellulase and xylanase production.

In this study, the transcriptomes of $P$. oxalicum HP7-1 when cultured on different carbon sources were profiled to identify candidate regulators of its cellulolytic and xylanolytic genes. Three novel key regulatory genes that simultaneously regulate the expression of major cellulase and xylanase genes were detected and identified. The three novel regulatory genes were also found to regulate the expression of one another.

\section{Results}

Forty candidate regulators of cellulolytic gene expression were detected in $P$. oxalicum with transcriptomic profiling

To screen for candidate regulators of cellulolytic gene expression in $P$. oxalicum, deep RNA sequencing (RNAseq) was used to analyze the transcript profiles of $P$. oxalicum HP7-1. After medium containing glucose (Glu), wheat bran (WB), or wheat bran plus Avicel (WA) as the sole carbon source was directly inoculated with P. oxalicum HP7-1, the fungal cells showed significant difference in their cellulase and xylanase activities. In the presence of Glu, P. oxalicum HP7-1 underwent carbon catabolite repression, and its cellulase and xylanase activities were barely detectable. In the 3 days after inoculation, all the enzymatic activities of strain HP7-1, including those of FPase, endo-glucanase (CMCase), $p$-nitrophenyl- $\beta$-cellobiosidase (pNPCase), $p$-nitrophenyl$\beta$-glucopyranosidase (pNPGase), and xylanase, were similar on WB and WA. After day 3 post-inoculation, the FPase, CMCase, pNPCase, and xylanase activities on WA increased sharply compared with those on WB. The maximum FPase activities of strain HP7-1 were $1.45 \pm 0.01$ and $0.5 \pm 0.04 \mathrm{U} / \mathrm{mL}$ on WA and $\mathrm{WB}$, respectively, on day 6 after inoculation. Interestingly, the pNPGase activity of HP7-1 on WA was lower than that on WB between days 3 and 5 after inoculation, whereas after day 5 , it was higher on WA than on WB (Additional file 1: Figure S1). P. oxalicum HP7-1 also produced FPase activity of $0.24 \pm 0.07 \mathrm{U} /$ $\mathrm{mL}$ on Avicel on day 6 after inoculation.

Glucose is known to induce CCR. WB is a low-cost agricultural waste product, composed predominantly of starch ( 19\%), nonstarch polysaccharides $(\sim 58 \%)$, and crude protein $(\sim 18 \%)$. Among the nonstarch polysaccharides, soluble cello-oligosaccharides are the most significant factors in cellulase production in P. oxalicum [21]. Although WB and Avicel both induce cellulase production, $P$. oxalicum HP7-1 produced more cellulase when grown on WA than when grown on WB or Avicel alone. The cellulase and xylanase activities of $P$. oxalicum HP7-1 differed significantly when it was cultured on inducing or repressing carbon sources. Therefore, the transcriptomes of HP7-1 grown on WA, WB, and Glu were screened for candidate regulators of cellulase and xylanase gene expression.

The total RNAs were extracted from the mycelia of P. oxalicum HP7-1 grown on WA, WB, or Glu for $72 \mathrm{~h}$, and then sequenced. In total, approximately 26-27 million clean reads, $90 \mathrm{bp}$ in length (Accession Number SRA505232), were generated from each sample, constituting an average 80 -fold coverage of the $P$. oxalicum HP7-1 genome [5]. The average clean reads were mapped to 8623 predicted protein-coding genes in the HP7-1 genome (Additional file 2: Table S1). The Pearson's correlation coefficients were high $(R \geq 0.80)$ for the three biological replicates produced under each set of culture conditions (Additional file 3: Figure S2).

The clean reads obtained were then mapped onto the genome of P. oxalicum HP7-1 with the software BWA [22] and Bowtie [23]. The expression levels (fragments per kilobase of exon per million mapped reads, FPKM) of the genes were calculated with the software package RSEM [24], and the differentially expressed genes were detected with NOISeq in the R package [25]. Overall, a 
comparative analysis of these genes identified 1073, 667, and 491 genes as significantly differentially expressed between HP7-1_WB and HP7-1_Glu, HP7-1_WA and HP7-1_Glu, and HP7-1_WA and HP7-1_WB, respectively ( $\mid \log _{2}$ fold change $\mid \geq 0.8$ and probability $\geq 0.8$ were used as the thresholds). These gene sets contained 600,382 , and 242 upregulated genes, respectively, which mainly functioned in metabolic pathways, such as carbohydrate metabolism and energy metabolism (Fig. 1a, b). Among these differentially expressed genes, 108 were co-expressed on all the carbon sources tested (Additional file 4: Table S2). They formed three major groups with internally similar expression patterns in a heatmap hierarchical clustering assay: Group A containing 37 genes, Group B containing 37 genes, and Group C containing 34 genes (Fig. 1c).

Group A consisted of 37 genes displaying low expression (L) in Glu culture, moderate expression (M) in WB culture, and high expression $(\mathrm{H})$ in WA culture. Of these, 11 were cellulase genes, including two cellobiohydrolase genes (cbhs; POX04786/Cel6A and POX05587/Cel7A-2), seven endo- $\beta$-1,4-glucanase genes (egs; $P O X 01166 / \mathrm{Cel} 5 \mathrm{~B}$, POX01896/Cel5C, POX02740, POX05570/Cel45A, POX05571/Cel7B, POX06147/Cel5A, and POX06983), two $\beta$-glucosidase genes (bgls; POX03641 and POX06079). Two hemicellulase genes (POX01937 and POX03430), three genes encoding enzymes with predicted activity against polysaccharides (POX02308, POX08485, and $P O X 08897)$, and seven genes encoding major facilitator superfamily (MFS) members, including cellodextrin transporters (POX06051/CdtC and POX05915/CdtD [26]) were included (Fig. 1c). These data strongly support the finding that the strongest FPase activity $(1.45 \mathrm{U} / \mathrm{mL})$ in P. oxalicum HP7-1 was observed in WA culture (Additional file 1: Figure S1).

By screening the genome of $P$. oxalicum HP7-1, we detected 484 genes encoding predicted TFs [5]. Of these, 35,15 , and 9 encoded putative TFs and were differentially expressed in comparisons of HP7-1_WB and HP7-1_ Glu, HP7-1_WA and HP7-1_Glu, and HP7-1_WA and

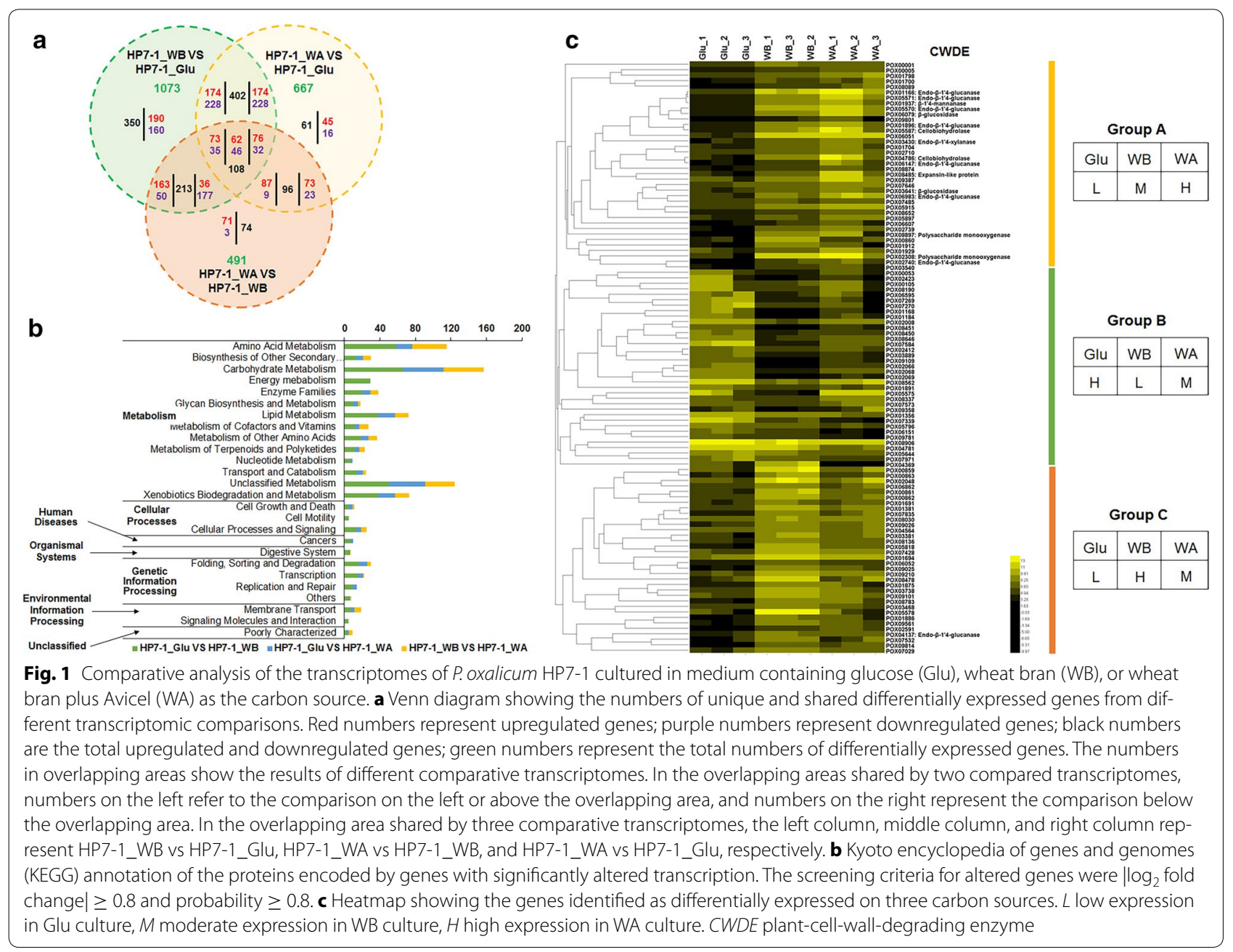


HP7-1_WB, respectively, when $\mid \log _{2}$ fold change $\mid \geq 1.5$ and probability $\geq 0.8$ were used as the thresholds. Of these, only POX01184 was differently expressed in all three comparisons. Eleven genes were shared in both comparisons HP7-1_WB vs HP7-1_Glu and HP7-1_WA vs HP7-1_Glu, and six were shared in both HP7-1_WB vs HP7-1_Glu and HP7-1_WA vs HP7-1_WB (Fig. 2a). In total, 40 candidate genes encoding putative TFs were detected, which appeared at least once in all the comparisons of the transcriptomes expressed on the different carbon sources (WA, WB, and Glu). The expression of these genes was induced or repressed to varying degrees by the different carbon sources, ranging from $1.5<\mid \log _{2}$ fold change $\mid<6.25$ (Additional file 5: Table S3).
The proteins encoded by the candidate genes were classified into eight types, and about $55 \%$ of them contained a zinc finger structure and 15\% were homeodomain-like proteins. Seven candidates were homologues of known regulators identified in cellulolytic fungi, including six proteins that regulate cellulase and/or xylanase gene expression (POX01960/ ClrB [PoxClrB] [5], POX00972/ClrC [PoxClrC] [17], POX04860/PDE_07199 [17], POX06534/BrlA [PoxBrlA] [27], POX08522 [5], and POX02768/PacC [PoxPacC] [28]), and one protein (POX07099/FlbD [PoxFlbD] [29]) that regulates the expression of genes associated with fungal conidiation (Additional file 5: Table S3).

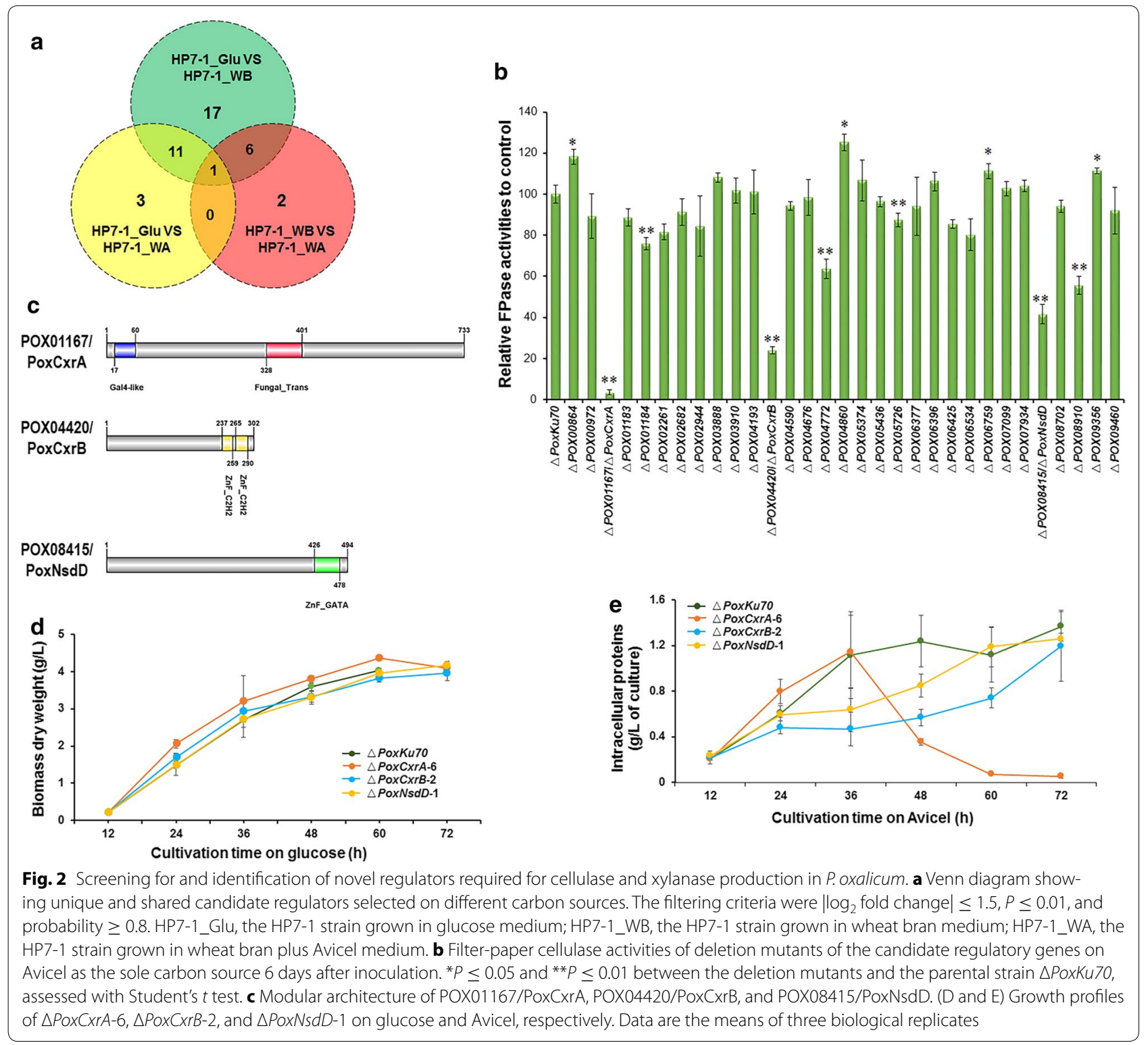


Ten novel regulators are required for cellulase production in $P$. oxalicum

We attempted to knock-out the 40 candidate genes in the mutant $\triangle P o x K u 70$ [5], which allows high-frequency gene deletion through homologous recombination. Pox$C l r B$ and POX08522 were previously knocked-out and identified as regulators of cellulase and xylanase genes when P. oxalicum HP7-1 was grown on Avicel [5]. Ultimately, 31 deletion mutants were constructed and confirmed with a PCR analysis (Additional file 6: Figure S3) using gene-specific primers (Additional file 7: Table S4), so the deletion of the target genes in $\triangle$ PoxKu 70 was $82 \%$ successful. Eleven mutants showed significantly altered FPase activities, ranging from 11.2 to $96.8 \%$ of the activity of the parental strain $\triangle P o x K u 70(P \leq 0.05$, Student's $t$ test; Fig. 2b). Of the genes deleted in these 11 mutants, 10 encoded regulators found to be involved in cellulase production in the filamentous fungi for the first time in this study: POX00864, POX01167, POX01184, POX04420, POX04772, POX05726, POX06759, POX08910, POX08415, and POX09356 (Table 1). The other gene, $P O X 04860$, encoded the protein homologue of PDE_07199 from P. oxalicum 114-2, which negatively affects cellulase production, as described previously [17]. Importantly, $\triangle P O X 01167$ lost almost all its FPase activity when grown on Avicel. $\triangle P O X 04420$ and $\triangle P O X 08415$ showed 75.9 and $58.5 \%$ reduction in FPase activity compared with the parental strain $\triangle P o x K u 70$ when grown on Avicel, respectively (Fig. 2b). These mutants ranked as the top three in the "hierarchy" of FPase activity, and were selected for further study. $\triangle P O X 01167, \triangle P O X 04420$, and $\triangle P O X 08415$ were also confirmed with a Southern hybridization analysis (Additional file 6: Figure S3) with specific probes (Additional file 7: Table S4) to exclude the possibility that multiple copies of the deletion cassette were inserted into the $\triangle$ PoxKu70 genome.

\section{PoxCxrA, PoxCxrB, and PoxNsdD are zinc finger TFs}

POX01167 encodes a polypeptide of 733 amino acids. A BLAST analysis at the National Center for Biotechnology Information (NCBI) showed that POX01167 contains a GAL4-like $\mathrm{Zn}_{2} \mathrm{Cys}_{6}$ binuclear cluster DNAbinding domain (cd00067) and a fungal TF regulatory middle homology region (cd12148) (Fig. 2c). POX01167 is conserved in P. oxalicum (i.e., 99\% identity with PDE_09277 [EPS34263.1] in P. oxalicum 114-2), and shares weak identity with proteins in other filamentous fungi that are capable of lignocellulosic degradation (i.e., 66\% with EN45_038550 [KZN93671.1] in P. chrysogenum P2niaD18, and 44\% with ANI_1_1528064 [XP_001391255.2] in A. niger CBS 513.88).

POX04420 encodes a polypeptide of 302 amino acids, which contains a two-zinc-finger double domain (pfam13456) (Fig. 2c). A protein alignment with BLAST showed that POX04420 is also conserved in P. oxalicum (i.e., 99\% identity with PDE_03425 [EPS28479.1] in $P$. oxalicum 114-2), but shares low identity with proteins in other cellulolytic fungi (i.e., 53\% with ANI_1_990104 [XP_001395874.1] in A. niger CBS 513.88].

Table 1 Regulatory genes detected in this study that regulate cellulase production in P. oxalicum HP7-1

\begin{tabular}{|c|c|c|c|c|c|c|}
\hline Gene ID & $\begin{array}{l}\text { GenBank } \\
\text { accession } \\
\text { number }\end{array}$ & $\begin{array}{l}\text { InterPro } \\
\text { annotation }\end{array}$ & Domain description & Known homologous TFs & $\begin{array}{l}\text { Identity } \\
(\%)\end{array}$ & $\begin{array}{l}\text { FPase activity } \\
\text { of mutant relative } \\
\text { to parent strain (\%) }\end{array}$ \\
\hline POX00864 & KY860734 & IPR001138 & Zinc finger, Zn2Cys6 type & NA & NA & $118.3 \pm 3.6$ \\
\hline POX01167 & KY368172 & $\begin{array}{l}\text { IPR001138 } \\
\text { IPR007219 }\end{array}$ & $\begin{array}{l}\text { Zinc finger, Zn2Cys6 type; } \\
\text { Fungal_Trans }\end{array}$ & NA & NA & $3.6 \pm 1.3$ \\
\hline POX01184 & KY860735 & |PR011991 & $\begin{array}{l}\text { Winged helix repressor } \\
\text { DNA-binding domain }\end{array}$ & NA & NA & $76.0 \pm 3.0$ \\
\hline POX04420 & KY368173 & IPR007087 & Zinc finger, $\mathrm{C} 2 \mathrm{H} 2$ type & NA & NA & $24.1 \pm 1.7$ \\
\hline POX04772 & KY860736 & IPR009071 & High mobility group box (HMG) & $\begin{array}{l}\text { A. nidulans FGSC A4 } \\
\mathrm{HmbB}\end{array}$ & 41 & $63.8 \pm 4.8$ \\
\hline POX04860 & KY922971 & IPR009057 & Homeodomain-like & $\begin{array}{l}\text { P. oxalicum 114-2 } \\
\text { PDE_07199 }\end{array}$ & 99 & $125.2 \pm 4.1$ \\
\hline POX05726 & KY860737 & IPR007087 & Zinc finger, $\mathrm{C} 2 \mathrm{H} 2$ type & NA & NA & $87.5 \pm 3.3$ \\
\hline POX06759 & KY860738 & IPR001138 & Zinc finger, Zn2Cys6 type & $\begin{array}{l}\text { Nectria haematococca mpVI } \\
\text { CTF1 beta }\end{array}$ & 34 & $111.2 \pm 3.8$ \\
\hline POX08415 & KY368171 & IPR000679 & Zinc finger, GATA type & A. nidulans FGSC A4 NsdD & 57 & $41.5 \pm 4.7$ \\
\hline POX08910 & KY860739 & $\begin{array}{l}\text { IPR009057 } \\
\text { IPR007526 }\end{array}$ & $\begin{array}{l}\text { Homeodomain-like; } \\
\text { SWIRM domain }\end{array}$ & NA & NA & $55.6 \pm 4.5$ \\
\hline POX09356 & KY860740 & IPR001138 & Zinc finger, Zn2Cys6 type & NA & NA & $111.3 \pm 1.4$ \\
\hline
\end{tabular}

IPR InterPro database (http://www.ebi.ac.uk/interpro/scan.html), TF transcription factor, NA not annotated, CTF1 beta cutinase transcription factor 1 beta, FPase filterpaper cellulase 
POX08415 is a 494-amino acid polypeptide, which contains a conserved GATA-type zinc-finger DNAbinding domain with the amino acid sequence $\mathrm{C}-\mathrm{X}_{2}-\mathrm{C}$ $\mathrm{X}_{18}-\mathrm{C}-\mathrm{X}_{2}-\mathrm{C}$ at its $\mathrm{C}$-terminus. A BlastP analyses showed that POX08415 shares 99\% identity with PDE_02029 (EPS27088.1) in P. oxalicum 114-2, and $57-64 \%$ identity with NsdD in $A$. nidulans FGSC4 (XP_660756.1), $A$. flavus NRRL3357 (XP_002376041.1), and A. fumigatus AF293 (XP_754237.1).

In subsequent analyses, POX01167 and POX04420 were redesignated PoxCxrA (cellulolytic and xylanolytic regulator A in P. oxalicum) and PoxCxrB, respectively. POX08415 was renamed PoxNsdD (NsdD in P. oxalicum) based on the name of its homolog NsdD in Aspergillus.

A neighbor-joining phylogenetic tree indicated that PoxCxrA and PoxCxrB of P. oxalicum are closely related to the corresponding homologues in other cellulolytic fungi, including Aspergillus sp. and Talaromyces sp., but not to those in T. reesei or N. crassa, whereas PoxNsdD is closely related to the homologues in $T$. reesei, $N$. crassa, and Talaromyces sp., but not to that in Aspergillus sp. (Additional file 8: Figure S4).

\section{Growth, cellulase and xylanase production of $\triangle P o x C x r A$, $\triangle P o x C x r B$, and $\triangle P o x N s d D$ were impaired on Avicel}

To determine whether these proteins affect $P$. oxalicum cell growth, the growth of $\triangle P o x C x r A$ ( $\triangle P O X 01167)$, $\triangle P o x C x r B(\triangle P O X 04420)$, and $\triangle P o x N s d D(\triangle P O X 08415)$ was compared with that of the parental strain $\triangle P o x K u 70$ when they were directly inoculated into medium containing glucose or Avicel as the sole carbon source. All three mutants accumulated similar amounts of mycelial biomass to that accumulated by $\triangle P o x K u 70$ in glucose medium (Fig. 2d), indicating that the knock-out of none of the three genes affected $P$. oxalicum growth when it utilized glucose as the carbon source.

In contrast, the mycelial intracellular proteins of $\triangle$ PoxCxrA were similar to those of $\triangle$ PoxKu 70 before culture for $36 \mathrm{~h}$ in Avicel medium, but dropped sharply to approximately a negligible level between 36 and $72 \mathrm{~h}$. $\triangle P o x N s d D$ produced a similar amount of intracellular protein to $\triangle P o x K u 70$ during culture for $24 \mathrm{~h}$, but after that time, the amount began to decrease relative to that produced by $\triangle$ PoxKu 70 . Notably, by $60 \mathrm{~h}$, the amount of intracellular protein in $\triangle P O x N s d D$ was similar to that in $\triangle P o x K u 70$. Moreover, $\triangle P o x C x r B$ contained less mycelial intracellular protein than either $\triangle P o x K u 70$ or $\triangle P o x N s d D$ during the whole culture period (Fig. 2e). These results clearly indicate that PoxCxrA, PoxCxrB, and PoxNsdD are required for fungal growth on Avicel.

Based on the growth of $\triangle P o x C x r A$ after its direct inoculation into Avicel medium (Fig. 2e), we hypothesized that $\triangle$ PoxCxrA might not respond to complex and recalcitrant carbon sources. To test this hypothesis, we first investigated $\triangle P o x C x r A$ growth during a shift experiment from glucose to Avicel. The growth of $\triangle P o x C x r A$ was impaired (Fig. 3a) and the activities of its extracellular cellulase and xylanase were significantly reduced by the shift (Fig. 3b-f).

A shift experiment from glucose to Avicel was used to investigate the cellulase and xylanase production by $\triangle P o x C x r B$ and $\triangle P o x N s d D$ in the presence of Avicel. The production of FPase, CMCase, pNPCase, and xylanase by the two deletion mutants was significantly lower than that of $\triangle$ PoxKu70 ( $P \leq 0.05$, Student's $t$ test) (Fig. $4 \mathrm{a}-$ d). Interestingly, the pNPGase activity in the mutant $\triangle P o x N s d D$ increased on average by $217-293 \%$, whereas it only increased by $53.2 \%$ in the mutant $\triangle P o x C x r B$ on day 5 after induction $(P \leq 0.01$, Student's $t$ test) compared with the activity in $\triangle$ PoxKu 70 (Fig. 4e).

To further verify that the changes in cellulase and xylanase production by the mutants $\triangle P o x C x r A$, $\triangle P o x C x r B$ and $\triangle P o x N s d D$ resulted from the specific deletion of the corresponding gene, the complemented strain of each of the three mutants was constructed, confirmed by PCR (Additional file 7: Table S4 and Additional file 9: Figure S5), and then was tested for enzyme production when grown on Avicel for 4 days after a transfer from glucose to Avicel as described in "Methods" section. The results showed that the complemented strain $C \triangle P o x C x r A$ could produce $51-74.6 \%$ of cellulase activities and $28.2 \%$ of xylanase activity of the parent strain $\triangle$ PoxKu 70 , which were significantly higher than those of the mutant strain $\triangle \operatorname{Pox} C x r A(P \leq 0.01$, Student's $t$ test; Fig. 5a-e). Moreover, the complemented strains $C \triangle P o x C x r B$ and $C \Delta P o x N s d D$ produced $49.1-$ 73.5\% of cellulase activities except for pNPGase activity and $\sim 81 \%$ of xylanase activity of the $\triangle P o x K u 70$, which were also significantly higher than those of $\triangle P o x C x r B$ and $\triangle P o x N s d D(P \leq 0.05$, Student's $t$ test; Fig. $5 \mathrm{a}-\mathrm{c}, \mathrm{e})$. The pNPGase activity of $\mathrm{C} \triangle P o x C x r B$ and $C \triangle P o x N s d D$ was 156.6 and $308.0 \%$ of that of $\triangle P o x K u 70$, respectively, and was significantly lower than that of $\triangle P o x C x r B$ and $\triangle$ PoxNsdD ( $P \leq 0.05$, Student's $t$ test; Fig. $5 \mathrm{~d})$. These data confirmed that the alterations in cellulase and xylanase production by the mutants $\triangle$ PoxCxrA, $\triangle$ PoxCxrB and $\triangle P o x N s d D$ resulted from the deletion of genes PoxCxrA, PoxCxrB and PoxNsdD.

\section{Quantitative reverse transcription (RT)-PCR shows that PoxCxrA, PoxCxrB, and PoxNsdD regulate one another and the expression of cellulase and xylanase genes}

To comprehensively analyze the regulatory functions of the identified regulators, mutants $\triangle P$ PoxC $x r A$, $\triangle P o x C x r B$, and $\triangle P o x N s d D$ were subjected to an RTqPCR analysis, using as the control $\triangle P o x K u 70$ grown 

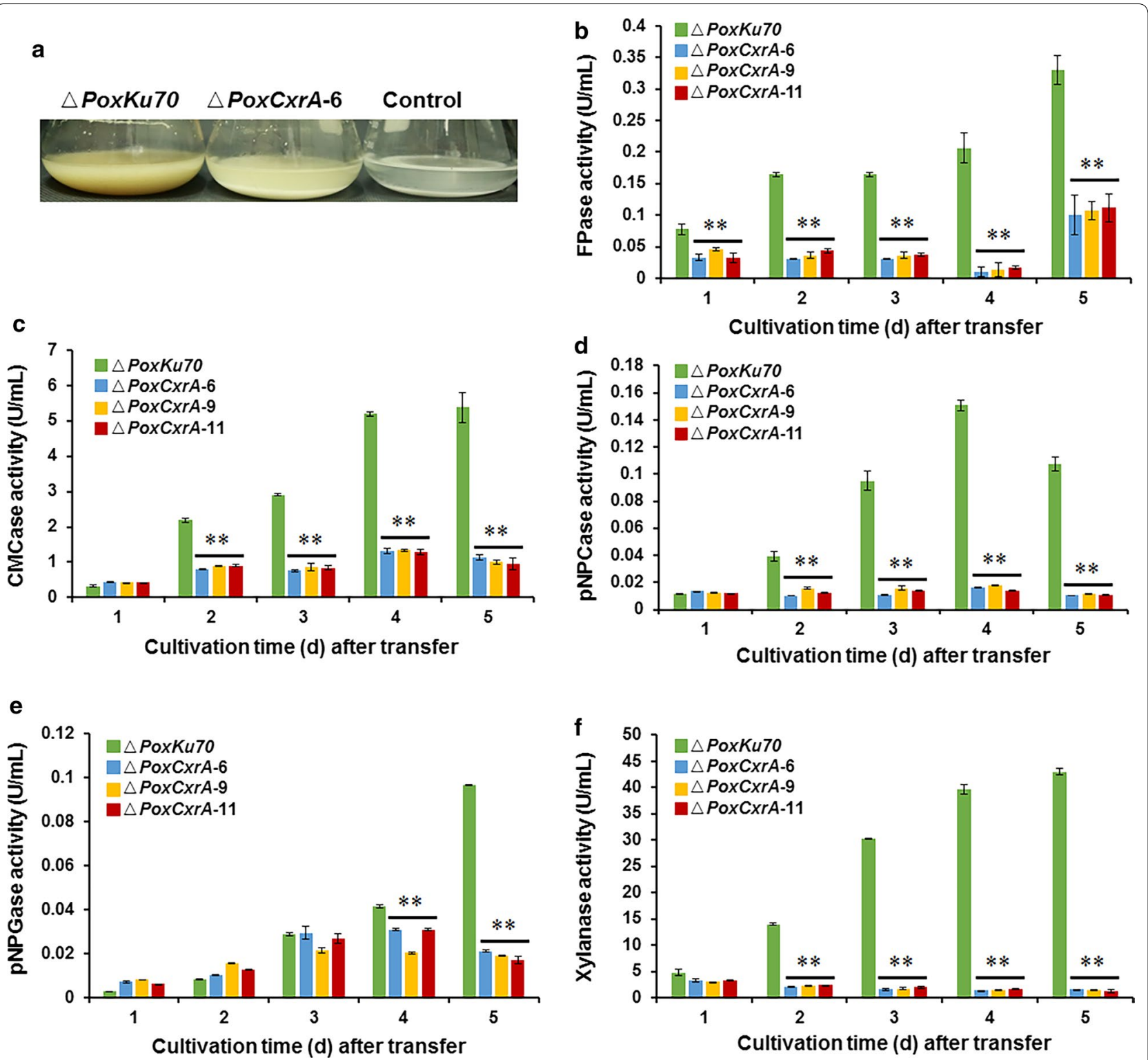

Fig. 3 Deletion of PoxCXrA abolished the utilization of cellulose by P. oxalicum. a Growth of $\triangle P O x C x r A$ and $\triangle P$ oxKu 70 on Avicel for 4 days after a shift from glucose; medium without inoculant was used as the blank control. (b-f) FPase, CMCase, pNPCase, pNPGase, and xylanase activities, respectively, of crude enzymes from $\triangle P O x C \times r A$ and $\triangle P O X K u 70$ after a shift from glucose to Avicel. Crude enzymes were produced by fungal strains cultured on 2\% Avicel as the sole carbon source. Enzyme activities were measured 1,2, 3, 4, and 5 days after the shift. Data are the means of three biological replicates. FPase filter-paper cellulase, CMCase carboxymethylcellulase, $p N P C$ ase $p$-nitrophenyl- $\beta$-cellobiosidase, $p N P G$ ase $p$-nitrophenyl- $\beta$ glucopyranosidase

under the same culture conditions. The transcription levels of the major cellulase and xylanase genes and PoxCxrA, PoxCxrB, and $\triangle P o x N s d D$ in $\triangle P o x C x r A$, $\triangle P o x C x r B$, and $\triangle P o x N s d D$ were measured $4,12,24$, and $48 \mathrm{~h}$ after the shift from glucose to Avicel (Fig. 6). The cellulase and xylanase genes tested included two cbhs (POX05587/Cel7A-2 and POX04786/Cel6A), seven egs (POX01166/Cel5B, POX02740, POX04137, POX05571/Cel7B, POX06147/Cel5A, POX06983, and POX07535/Cel12A), one bgl (POX06835/Bgl3A), and three xyns (POX05916, POX06783/Xyn11A, and POX08484/Xyn11B).

In $\triangle P o x C x r A$, the transcripts of almost half the cellulase and xylanase genes were significantly downregulated to $26.0-87.8 \%$ at $4 \mathrm{~h}$ of induction, whereas the remaining genes were upregulated by $50.0-341.4 \%(P \leq 0.05$, Student's $t$ test). After $4 \mathrm{~h}$, the transcription of almost all the genes had decreased by $22.3-99.5 \%(P \leq 0.05$, Student's $t$ test) in $\triangle P o x C x r A$ compared with that in the parental strain $\triangle$ PoxKu70 (Fig. 6). 

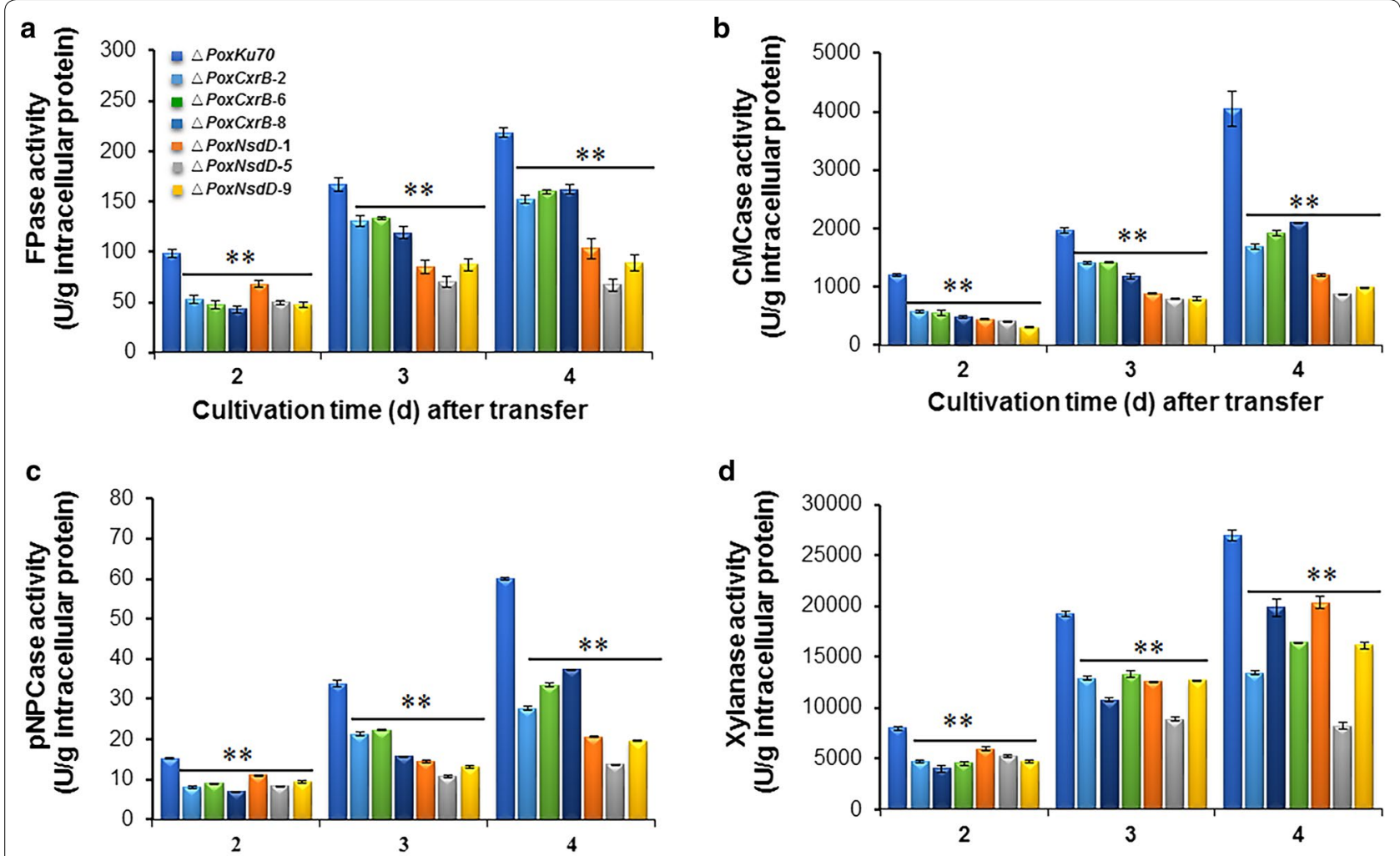

d

Cultivation time (d) after transfer
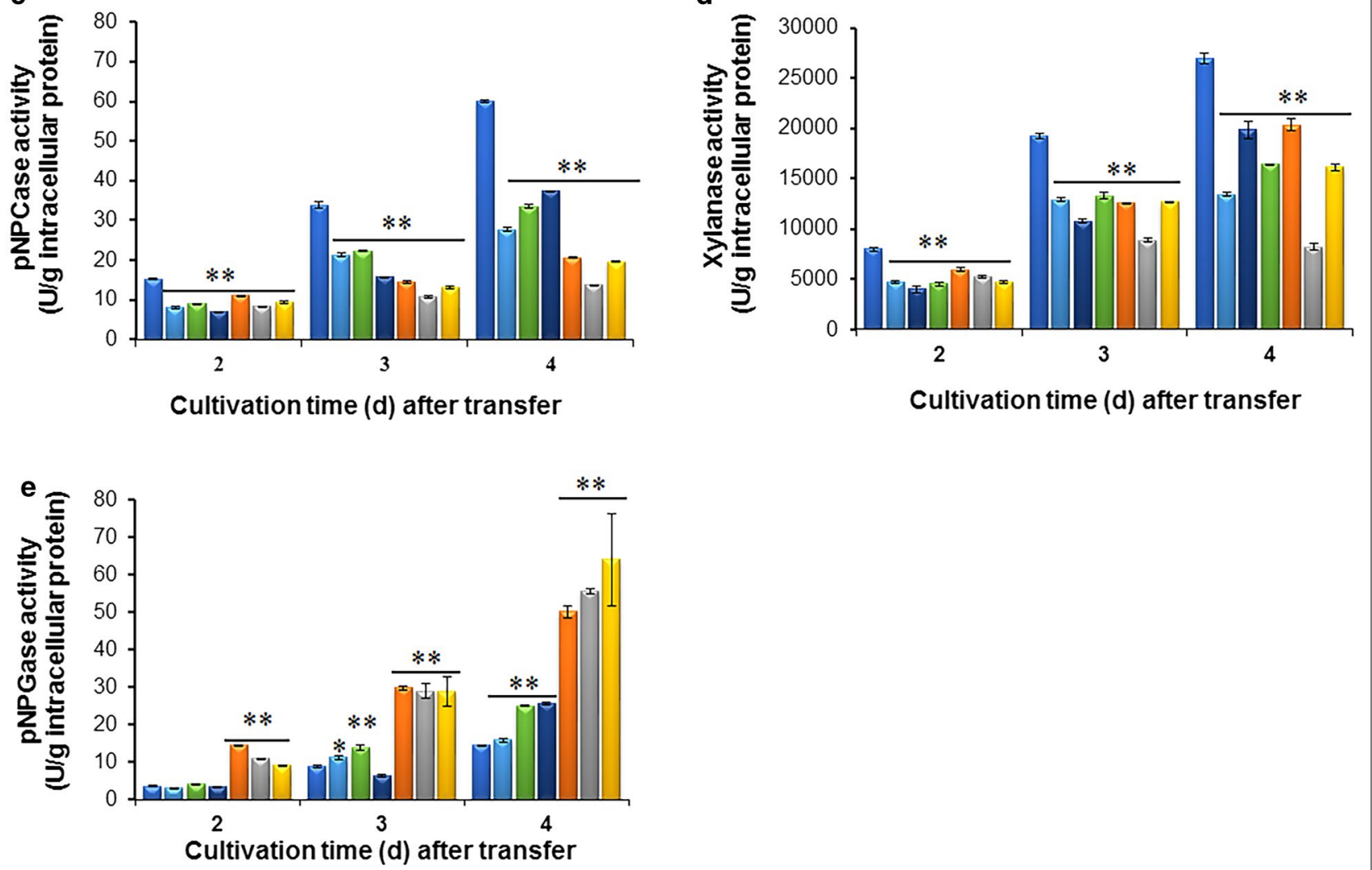

Fig. 4 Activities of crude enzymes from $\triangle P O x C x r B, \triangle P O x N s d D$, and $\triangle P o x K u 70$ after a shift from glucose to Avicel. Crude enzymes were produced by fungal strains grown in $2 \%$ Avicel as the sole carbon source. Enzymatic activity was measured 2, 3, and 4 days after the shift. ${ }^{*} P \leq 0.05$ and ${ }^{* *} P \leq 0.01$ between the regulatory gene mutants and the parental strain $\triangle P O x K u 70$, assessed with Student's $t$ test. a Filter-paper cellulase (FPase) activity. b Endo-glucanase (CMCase) activity. $\mathbf{c} p$-Nitrophenyl- $\beta$-cellobiosidase (pNPCase) activity. d Xylanase activity. e $p$-Nitrophenyl- $\beta$ glucopyranosidase (pNPGase) activity

In $\triangle P o x C x r A$, the transcripts of the PoxCxrB gene were downregulated to some extent (by $41.2-70.6 \%$ ) after $4 \mathrm{~h}$ of induction on Avicel ( $P \leq 0.05$, Student's $t$ test). These effects became more severe as the induction time increased. The transcripts of PoxNsdD in $\triangle P o x C x r A$ were upregulated by $63.6 \%$ at $24 \mathrm{~h}$, and then downregulated by $86.5 \%$ at $48 \mathrm{~h}(P \leq 0.01$, Student's $t$ test $)$ compared with those in the parental strain $\triangle$ PoxKu70 (Fig. 6).
The expression of cellulase and xylanase genes in $\triangle P o x C x r B$ and $\triangle P o x N s d D$ was also investigated. Almost all the genes tested were downregulated in $\triangle P o x C x r B$ and $\triangle P o x N s d D$ during the whole induction period. Notably, the expression of the e.g. gene POX06983 increased by 82.2 and $212.0 \%$ in the $\triangle P o x C x r B$ and $\triangle P o x N s d D$ mutants, respectively, after induction for $4 \mathrm{~h}$, and the expression of an important $x y n$ gene, POX08484/Xyn11B, 

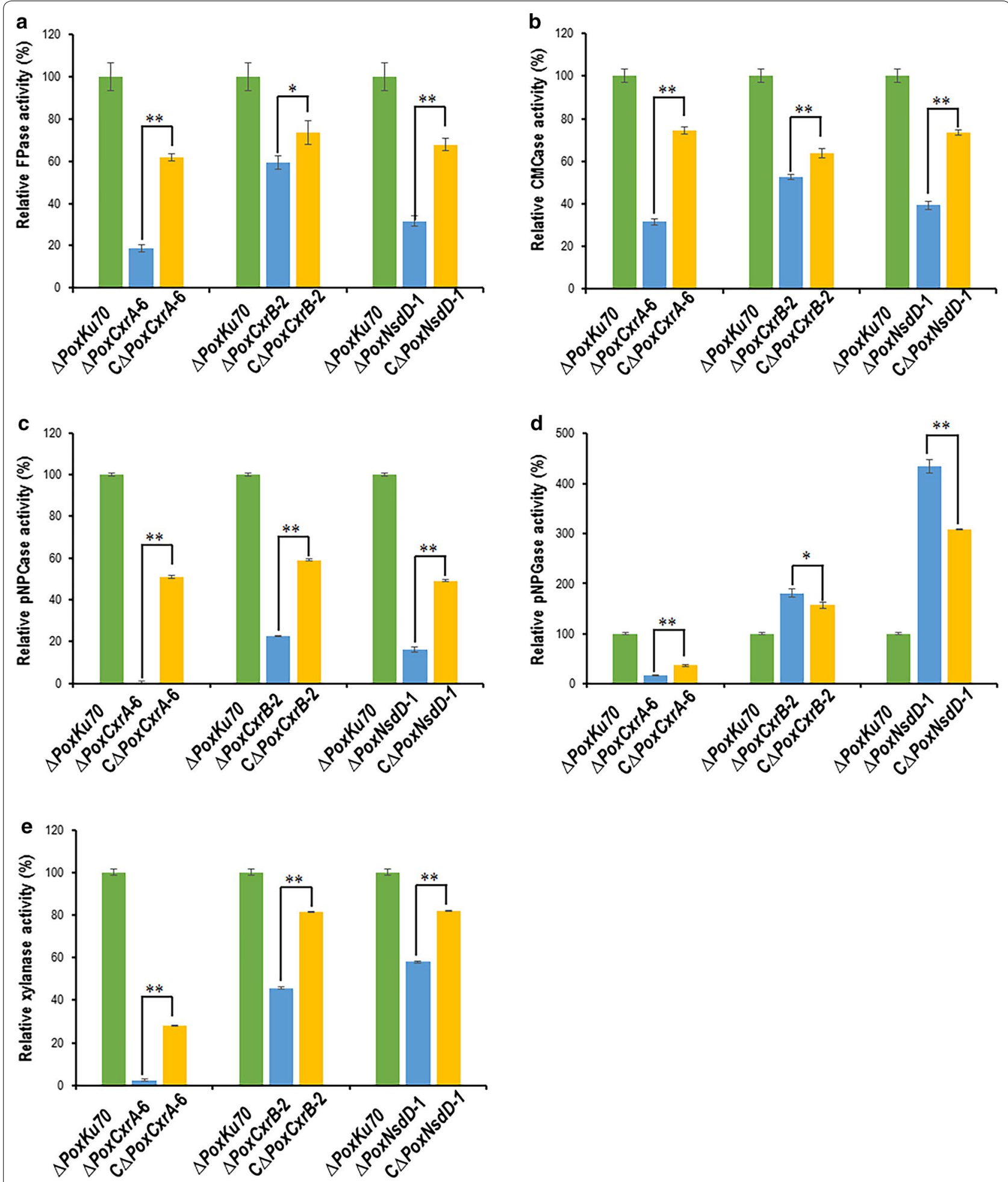

Fig. 5 Activities of crude enzymes from the complementary strains $C \triangle P o x C \times r A, C \triangle P o x C \times r B$ and $C \triangle P$ P X Ns $d D$ after a shift from glucose to Avicel. Crude enzymes were produced by fungal strains grown in $2 \%(\mathrm{~W} / \mathrm{v})$ Avicel as the sole carbon source for 4 days after the shift. ${ }^{* *} P \leq 0.01$ between the complementary strains and the regulatory gene mutants, assessed with Student's $t$ test. ${ }^{*} P \leq 0.05$ between $C \triangle P$ oxC $X r B$ and $\triangle P$ oxC $x r B$ assessed with Student's $t$ test. a Filter-paper cellulase (FPase) activity. b Endo-glucanase (CMCase) activity. $\mathbf{c} p$-Nitrophenyl- $\beta$-cellobiosidase (pNPCase) activity. d $p$-Nitrophenyl- $\beta$-glucopyranosidase (pNPGase) activity. e Xylanase activity 

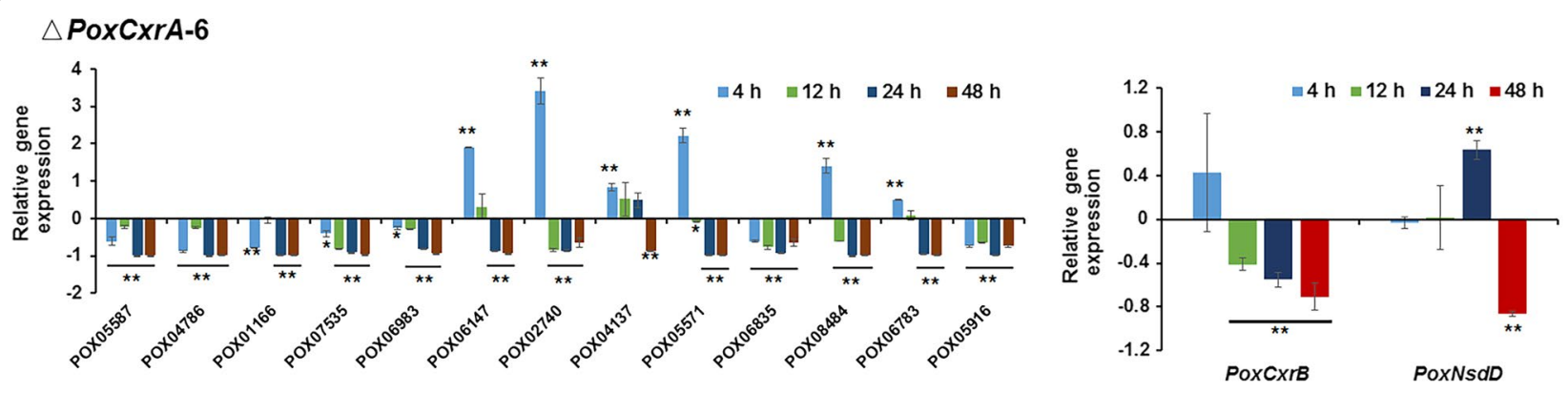

$\triangle \operatorname{PoxCxrB-2}$
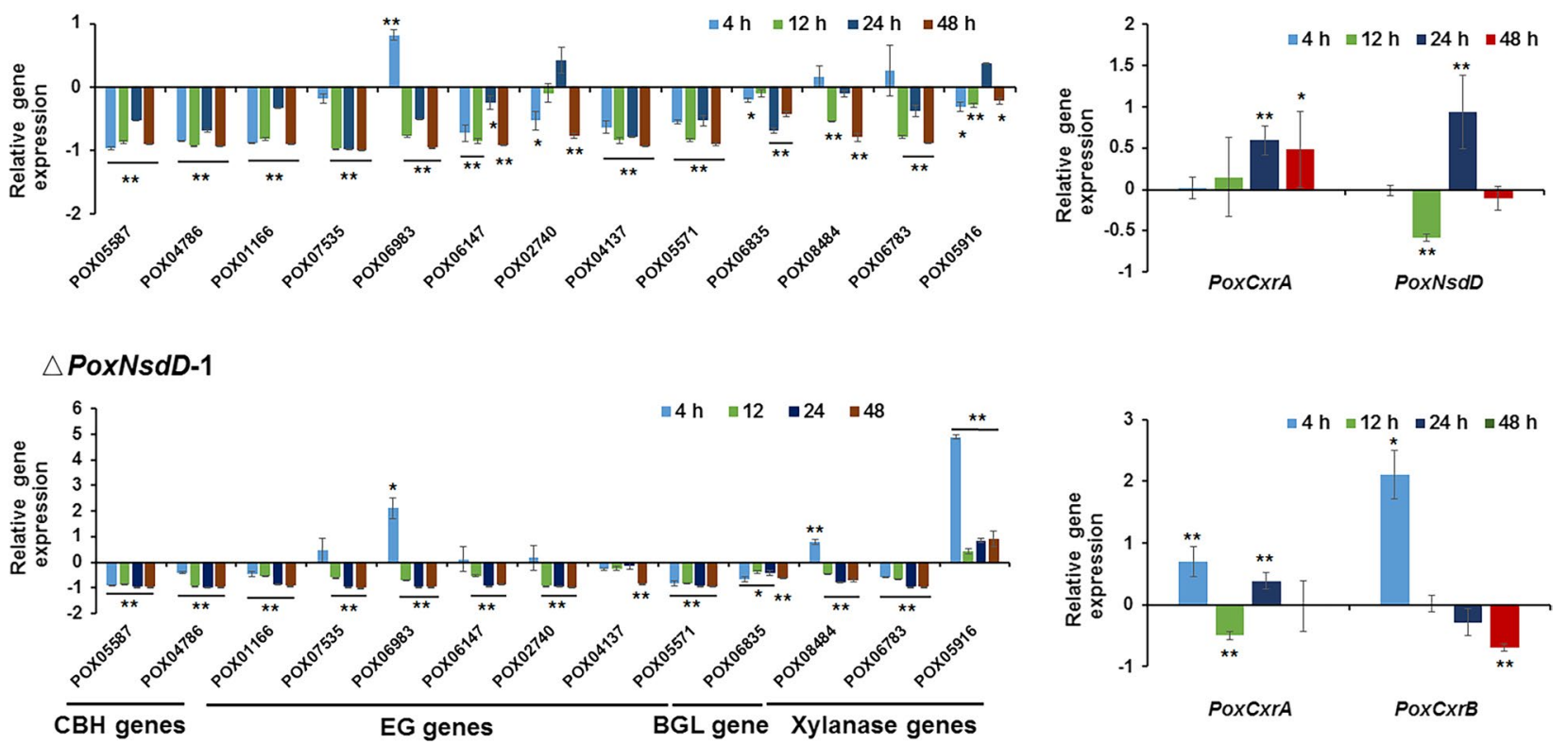

Fig. 6 Regulation of gene expression by $P$ ox $C x r A, P O x C x r B$, and PoxNsdD. Expression levels of cellulase and xylanase genes and the newly identified regulatory genes in the $\triangle P O x C \times r A-6, \triangle P O X C x r B-2$, and $\triangle P O x N s d D-1$ mutants at four different time points $(4,12,24$, and $48 \mathrm{~h})$ after the shift from glucose to Avicel, determined with RT-qPCR. Relative expression on the $y$ axis is the difference value for the transcription of the genes in the deletion mutants. ${ }^{*} P \leq 0.05$ and ${ }^{*} P \leq 0.01$ between the samples and $\triangle P O X K u 70$, assessed with Student's $t$ test. All experiments were performed independently in triplicate at least

was upregulated by $79.1 \%$ in mutant $\triangle P o x N s d D$ $(P \leq 0.05$, Student's $t$ test). The expression of another $x y n$ gene, POX05916, was also negatively regulated by $43.0-$ 489.7\% by PoxNsdD during the whole induction period on Avicel ( $P \leq 0.05$, Student's $t$ test; Fig. 6).

When the expression levels of PoxCxrA and PoxNsdD were tested in the mutant $\triangle P o x C x r B$, the transcripts of PoxCxrA were 59.2 and $48.8 \%$ higher than that in $\triangle P$ PoxKu70 after $24 \mathrm{~h}$ and $48 \mathrm{~h}$ of induction $(P \leq 0.05$, Student's $t$ test), respectively. However, PoxNs $d D$ expression first decreased by $58.2 \%$ at $12 \mathrm{~h}$, but subsequently increased by $93.4 \%$ at $24 \mathrm{~h}(P \leq 0.01$, Student's $t$ test; Fig. 6).

In $\triangle P o x N s d D$, the transcripts of PoxCxrA increased by 69.7 and $38.2 \%$ after 4 and $24 \mathrm{~h}$ of induction, respectively, whereas they decreased by $50 \%$ after $12 \mathrm{~h}$. Interestingly,
PoxCxrB expression in $\triangle P o x N s d D$ increased by $210.6 \%$ after induction for $4 \mathrm{~h}$, whereas after $48 \mathrm{~h}$, it had decreased by $69.3 \%$ compared with that in $\triangle$ PoxKu 70 $(P \leq 0.05$, Student's $t$ test; Fig. 6).

\section{PoxCxrA, PoxCxrB, and PoxNsdD bind to the promoter regions of targeted genes in vitro}

To confirm the regulation of the genes targeted by PoxCxrA, PoxCxrB, and PoxNsdD, in vitro binding experiments were performed with an electrophoretic mobility shift assay (EMSA). The thioredoxin (TRX)-His-Stagged putative DNA-binding domains of PoxCxrA, PoxCxrB, and PoxNsdD, designated PoxCxrA $17-150$, PoxCxrB $181-330$, and PoxNsdD ${ }_{335-494}$, respectively, were individually recombinantly expressed in Escherichia coli Rossetta, and purified. The putative protein-binding 
DNA fragments $(100-500 \mathrm{bp})$ in the promoter regions of the targeted genes, including the major cellulase and xylanase genes POX05587/Cel7A-2, POX01166/ Cel5B, POX06835/Bgl3A, and POX06783/Xyn11A, were tagged with 6-carboxyfluorescein (FAM). Shifted bands appeared in all the EMSA gels when PoxCxrA $\mathrm{A}_{17-150}$, PoxCxrB ${ }_{181-330}$, or PoxNsdD ${ }_{335-494}$ was mixed individually with the FAM-tagged DNA fragments corresponding to the promoter regions of these genes. The binding strengths increased dramatically as the amount of protein was increased from 1.2 to $4.8 \mu \mathrm{g}$ (Additional file 10: Figure S6). Competitive experiments were also performed using protein-binding DNA fragments lacking the FAM label as competitive probes. The concentrations of the shifted bands gradually decreased as the amount of competitive probe increased. However, no shifted band was detected between any DNA fragment and the high-concentration TRX-His-S fusion protein purified from the total proteins of $E$. coli cells containing the empty vector or bovine serum albumin (BSA) protein, which were used as the negative controls (Fig. 7). These results suggest that PoxCxrA $17-150$, PoxCxrB $181-330$, and PoxNsdD ${ }_{335-494}$ specifically bound all the tested DNA sequences from the promoter regions of the major cellulase and xylanase genes in vitro.

EMSA experiments were also performed to evaluate the interactions between the newly identified regulatory genes. The promoter regions of genes $\operatorname{Pox} C x r B$ and PoxNsdD were used as the probes to test their binding by PoxCxrA $\mathrm{A}_{17-150}$; those of PoxCxrA and PoxNsdD were used to test their binding by $\operatorname{PoxCxrB}{ }_{181-330}$; and those for PoxCxrA and PoxCxrB were used to test their binding by PoxNsdD $\mathrm{D}_{335-494}$. PoxCxrA $\mathrm{A}_{17-150}$, PoxCxrB ${ }_{181-330}$, and PoxNs $\mathrm{dD}_{335-494}$ specifically bound all the tested DNA sequences from the promoter regions of the newly identified regulatory genes in vitro (Fig. 8 and Additional file 11: Figure S7).

\section{Discussion}

In this study, 10 novel regulators of cellulase and xylanase gene expression in $P$. oxalicum were identified with transcriptomic profiling and genetic analyses. Among the 10 novel genes, the deletion mutants of PoxCxrA, Pox$C x r B$, and PoxNsdD genes were confirmed by PCR using gene-specific primers, Southern hybridization analysis and gene complementation. The cellulase and xylanase production of the complemented strains $C \triangle P o x C x r A$, $\mathrm{C} \triangle$ Pox $C x r B$ and $C \triangle P o x N s d D$ could not be fully restored to the levels of the parent strain $\triangle P o x K u 70$, which might result from different transcription levels of the complementary genes from that in $\triangle P o x K u 70$. The complementary genes PoxCxrB and PoxNsdD were introduced into the locus of an aspartic protease gene PoxPepA
(POX05007) whose deletion did not cause any alterations in cellulase and xylanase production in $\triangle$ PoxKu 70 (Jia-Xun Feng et al., unpublished), while the complementary cassette of PoxCxrA might randomly integrate into unknown locus in the genome. The knock-out of genes PoxCxrA, PoxCxrB, and PoxNsdD did not affect the vegetative growth of $P$. oxalicum in the presence of glucose, but very significantly affected the utilization of cellulose, indicating their important roles in cellulose degradation. The functions of PoxCxrA and PoxCxrB have not been reported in the literature until now.

PoxNsdD is a homologue of NsdD in Aspergillus. NsdD functions as an activator of sexual development [30] and a key repressor of asexual development in Aspergillus in specific environments [29, 31]. NsdD represses conidiation by downregulating the expression of $B r l A$, which encodes a key TF essential for conidiophore development in Aspergillus [30]. NsdD also functions downstream from fluffy gene G (FluG), but upstream from BrlA. In P. oxalicum 114-2, FluG deletion results in neither the fluffy phenotype nor any change in conidiation, and $B r l A$ is required but not sufficient for conidiation, suggesting that the mechanism of conidiophore development differs in P. oxalicum and $A$. nidulans [27]. However, NsdD has not yet been ascribed any regulatory function regarding cellulase and xylanase gene expression in the filamentous fungi.

Interestingly, in P. oxalicum HP7-1, the deletion of PoxCxrB and PoxNsdD reduced the production of $\mathrm{CBH}$, endo- $\beta-1,4$-glucanase (EG), and xylanase, but increased BGL production. BGL is responsible for the degradation of cellobiose to glucose, and cellobiose is a major inducer of cellulase gene expression. Therefore, an increase in BGL activity might reduce the amount of cellobiose, thus reducing the expression of the cellulase genes. Surprisingly, in $\triangle P o x C x r B$ and $\triangle P o x N s d D$, the transcription level of the $b g l$ gene POX06835/Bgl3A, which is thought to encode a predominantly extracellular BGL [32], was downregulated relative to its expression in $\triangle$ PoxKu 70 . However, the total extracellular BGL activities in the mutants were higher than that in $\triangle P o x K u 70$, suggesting that other extracellular BGLs exist.

The knock-out of the known regulatory gene $\mathrm{PoxClrC}$ caused no significant changes in FPase activity 6 days after inoculation onto Avicel compared with that in the parental strain $\triangle$ PoxKu 70 . However, its deletion significantly affected EG and/or BGL production (Jia-Xun Feng et al., unpublished observation). In a previous study, the BGL deletion mutant showed reduced cellulase production when cultured on Avicel medium after transfer from glucose medium [28]. These discrepancies may be attributable to different genetic backgrounds, different fungal culture conditions, and/or different methods of measuring enzyme production in the different studies. 


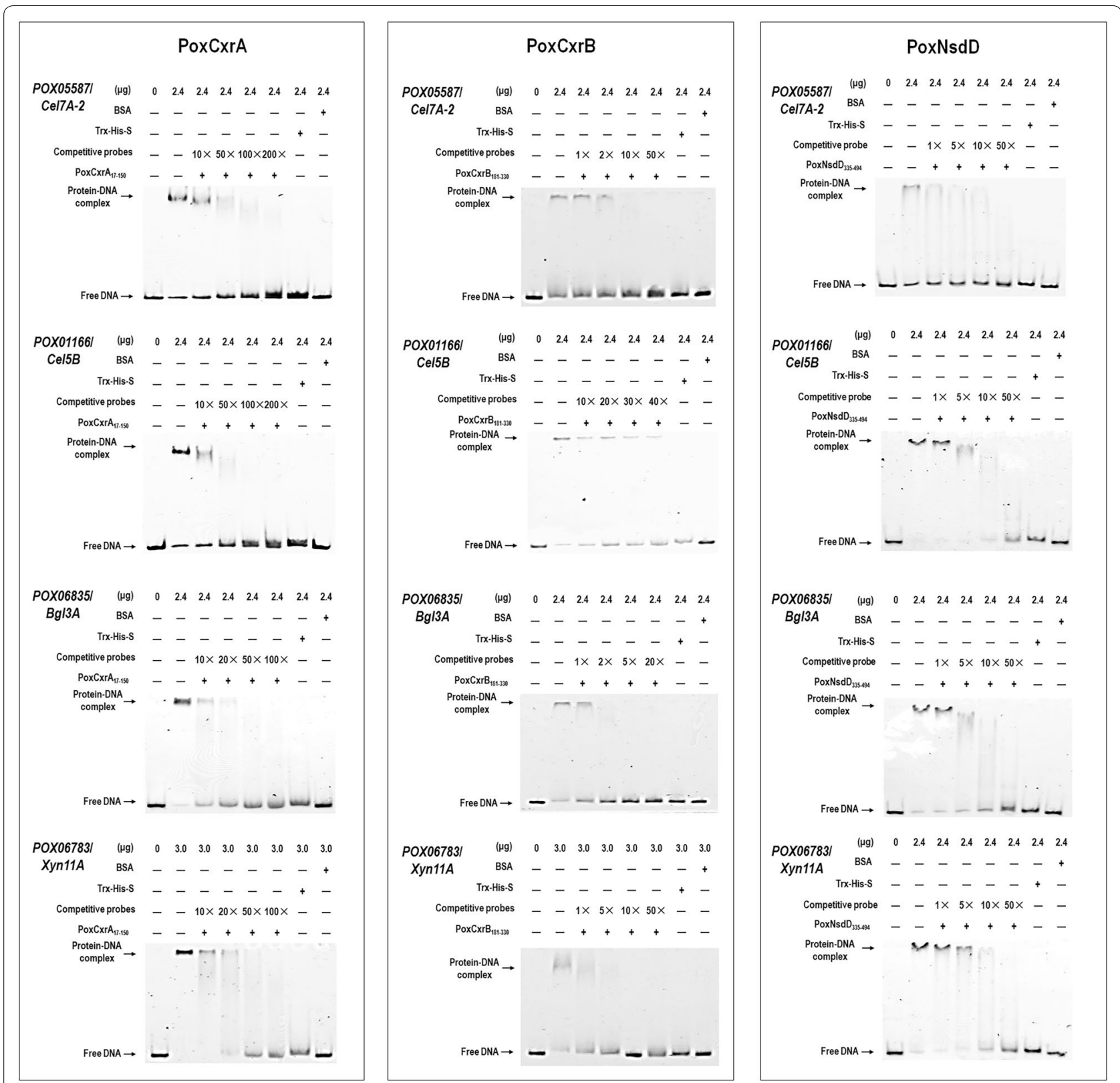

Fig. 7 Electrophoretic mobility shift assay showing the interactions between the DNA-binding domains of the regulators and the promoter sequences of cellulase and xylanase genes

Based on the experimental data generated in this study, we propose a novel real-time regulatory network of genes that controls the expression of the cellulase and xylanase genes of $P$. oxalicum in the presence of cellulose (Fig. 9). In the early stage of induction (4-12 h) of cellulose, PoxNsdD represses the expression of PoxCxrA and PoxCxrB. Subsequently, PoxNsdD stimulates PoxCxrA expression, and PoxCxrA induces PoxCxrB expression. Interestingly, $\operatorname{Pox} C x r B$ also enhances the transcription of PoxNsdD. By contrast, the regulatory relationships between these novel regulatory genes become more complex in the later period (24-48 h) of induction. At $24 \mathrm{~h}$, $\operatorname{Pox} C x r A$ increases the expression of $\operatorname{Pox} C x r B$, and suppressing the expression of $\operatorname{Pox} N s d D$, whose expression is also repressed by both $P o x C x r B$ and $P o x N s d D$. Moreover, PoxCxrB reduces the transcription of PoxNsdD. As at $24 \mathrm{~h}, \operatorname{Pox} C x r A$ increases the transcription of genes Pox$C x r B$ and $\operatorname{PoxNs} d D$, whose expression is repressed by PoxC $x r B$ at $48 \mathrm{~h}$, whereas PoxNsdD increases the expression of PoxCxrB at this time (Fig. 9). 


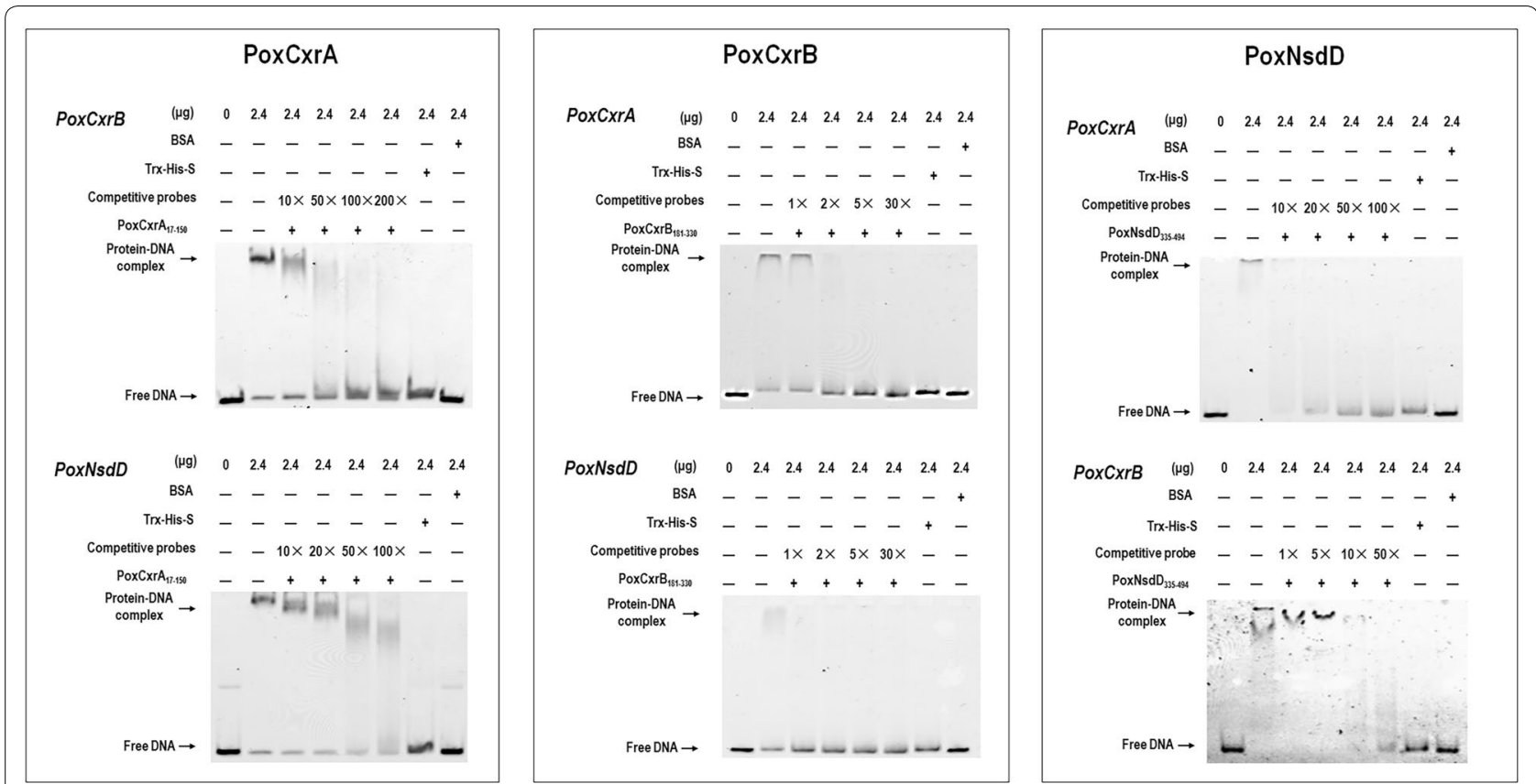

Fig. 8 Electrophoretic mobility shift assay showing the interactions between the DNA-binding domains of the regulators and the promoter sequences of the newly identified regulatory genes

During the whole period of induction, all three novel regulatory genes $\operatorname{Pox} C x r A, \operatorname{Pox} C x r B$, and PoxNsdD play positive roles in regulating the expression of the major cellulase and xylanase genes, including POX05587/Cel7A-2, POX01166/Cel5B, $P O X 06835 / B g l 3 A$, and POX06783/Xyn11A, except for the regulation of $P O X 01166 / C e l 5 B, P O X 06783 / X y n 11 A$, and POX06835/Bgl3A by PoxCxrA and PoxCxrB at 4 or $12 \mathrm{~h}$. Overall, the newly identified regulatory genes $P o x$ CxrA, PoxCxrB, and PoxNsdD regulate the expression of one another and directly regulate the expression of the cellulase and xylanase genes. However, the regulatory mechanism changes dynamically during the growth of fungal cells in the presence of cellulose (Fig. 9).

A protein alignment indicated that the three newly identified regulators, PoxCxrA, PoxCxrB, and PoxNsdD, are conserved in most filamentous ascomycete fungi capable of degrading cellulose, such as Aspergillus, Talaromyces, Trichoderma, and Neurospora. These data suggest that the three proteins and their homologues might play important roles in the regulation of cellulase and xylanase gene expression in ascomycete fungi, but this idea requires further confirmation.

\section{Conclusions}

In this study, our data show that PoxCxrA, PoxCxrB, and PoxNsdD are essential genes in the regulation of cellulase and xylanase gene expression in P. oxalicum. More importantly, PoxCxrA, PoxCxrB, and PoxNsdD regulate the expression of one another and directly regulate the expression of the cellulase and xylanase genes. These findings provide novel insights into the regulation of fungal cellulase and xylanase gene expression and provide a basis for genetically engineering or breeding fungi for the hyperproduction of cellulases and xylanases.

\section{Methods \\ Penicillium oxalicum strains and culture conditions}

The $P$. oxalicum strains used in this study were maintained on potato-dextrose agar (PDA) plates at $4{ }^{\circ} \mathrm{C}$. The fungal spores used for reproduction were harvested after 6 days on PDA plates at $28^{\circ} \mathrm{C}$, resuspended in buffer containing $0.1 \%$ Tween 80 , and adjusted to a concentration of $1 \times 10^{8}$ per milliliter.

For the transcriptomic (RNA-Seq) analysis of gene expression in P. oxalicum strain HP7-1 (China General Microbiological Culture Collection [CGMCC] no. 10781), $1 \mathrm{~mL}$ of spore suspension $\left(1.0 \times 10^{8} / \mathrm{mL}\right)$ was used to inoculate $100 \mathrm{~mL}$ of modified minimal medium (MMM [g/L]: $\left(\mathrm{NH}_{4}\right)_{2} \mathrm{SO}_{4} 4.0, \mathrm{KH}_{2} \mathrm{PO}_{4} 4.0, \mathrm{CaCl}_{2}$ 0.6, $\mathrm{MgSO}_{4} \cdot 7 \mathrm{H}_{2} \mathrm{O} 0.60, \mathrm{FeSO}_{4} \cdot 7 \mathrm{H}_{2} \mathrm{O} 0.005, \mathrm{MnSO}_{4} 0.0016$, $\mathrm{ZnCl}_{2}$ 0.0017, $\mathrm{CoCl}_{2} 0.002$, and $1 \mathrm{~mL}$ of Tween 80) containing glucose $(1.0 \mathrm{~g})$, wheat bran $(4.0 \mathrm{~g})$ (purchased from a local farmer's market in Nanning, China), or wheat bran (4.0 g) plus Avicel ${ }^{\circledR}$ PH-101 (2.0 g) (Cat No. 11365-1KG; Sigma-Aldrich, Darmstadt, Germany) as the 


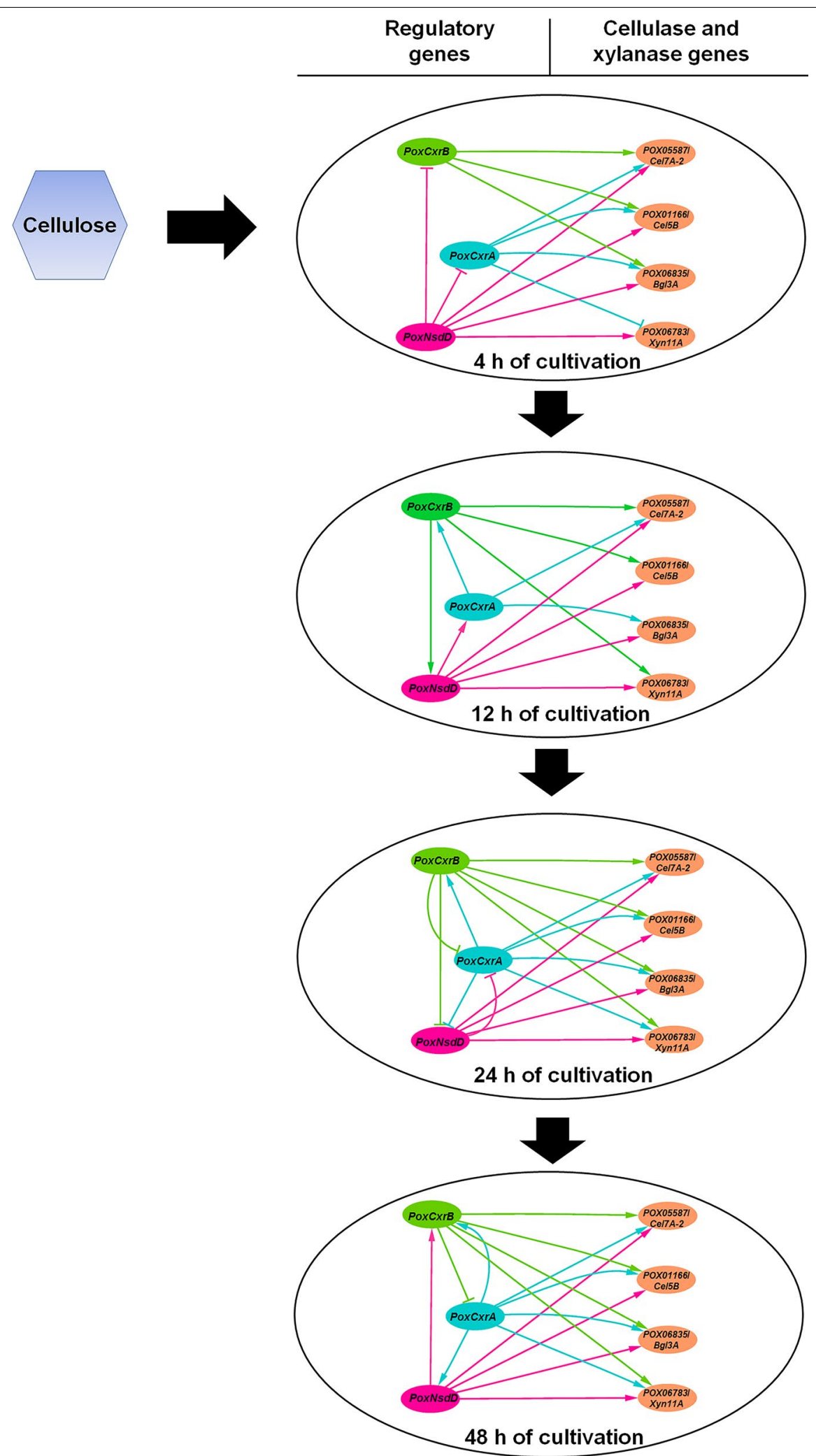

Fig. 9 Regulation network of the three novel key regulatory genes PoxC $x r A$, Pox $C x r B$, and PoxNsdD identified in P. oxalicum. Lines with arrows represent activation and barred lines represent inhibition. Green, blue, and purple lines show regulation by PoxCxrA, PoxCxrB, and PoxNsdD, respectively 
sole carbon source, respectively. The inoculated cultures were placed in a shaker at $28{ }^{\circ} \mathrm{C}$, and shaken at $180 \mathrm{rpm}$ for $72 \mathrm{~h}$. Mycelia were collected from the cultures with four-layer filter fabrics, and washed with diethyl-pyrocarbonate-treated water before RNA extraction.

The constructed $P$. oxalicum mutants of the candidate TF genes were inoculated into $100 \mathrm{~mL}$ of MMM containing a final concentration of $2 \%(\mathrm{w} / \mathrm{v})$ Avicel. The inoculated medium was incubated at $28{ }^{\circ} \mathrm{C}$, with shaking at $180 \mathrm{rpm}$ for 6 days. The supernatant was collected by centrifugation at $11,300 \times g$ for $10 \mathrm{~min}$ to assay the FPase activity.

To measure the growth profiles, $\triangle$ PoxCxrA

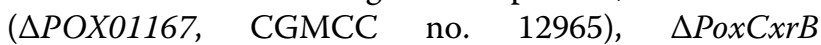
( $\triangle P O X 04420, \quad C G M C C$ no. 12966), $\triangle P o x N s d D$

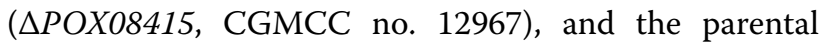
strain $\triangle P o x K u 70$ (CGMCC no. 3.15650) [5] were inoculated into MMM containing $1 \%(\mathrm{w} / \mathrm{v})$ glucose or $2 \%(\mathrm{w} / \mathrm{v})$ Avicel and incubated at $28{ }^{\circ} \mathrm{C}$ with shaking at $180 \mathrm{rpm}$. The mycelia were collected every $12 \mathrm{~h}$ until $72 \mathrm{~h}$.

A shift experiment was performed followed by an RTqPCR analysis of gene expression and an enzymatic activity assay of $\triangle P o x C x r A, \triangle P o x C x r B, \triangle P o x N s d D$, and the parental strain $\triangle P o x K u 70$ [5]. Approximately $1.0 \times 10^{8} /$ $\mathrm{mL}$ spores from the $P$. oxalicum mutant strains were grown in $100 \mathrm{~mL}$ of MMM containing $1 \%(\mathrm{w} / \mathrm{v})$ glucose at $28{ }^{\circ} \mathrm{C}$ with shaking at $180 \mathrm{rpm}$ for $24 \mathrm{~h}$. Equal portions of the harvested and washed mycelial samples were then aseptically inoculated into fresh MMM containing 2\% $(\mathrm{w} / \mathrm{v})$ Avicel as the sole carbon source. After culture for 4, 12,24 , or $48 \mathrm{~h}$, the mycelia were harvested, and the total RNA was extracted for RT-qPCR.

For the enzymatic activity assay of the three mutants $\triangle P o x C x r A, \triangle P o x C x r B$, and $\triangle P o x N s d D$ under induction conditions, the pregrown mycelia of these strains were cultured for 2-4 days in MMM containing Avicel as the sole carbon source, as described above.

\section{Total DNA and RNA extraction}

Total DNA and RNA were extracted with chemical methods, as previously described [5]. For total DNA extraction, the harvested mycelia were first washed with sterile water, ground in liquid nitrogen, and then added to a specific amount of lysate reagent $(40 \mathrm{mM}$ Tris- $\mathrm{HCl}, 10 \mathrm{mM}$ ethylenediaminetetraacetic acid, $20 \mathrm{mM}$ sodium acetate, and $1 \%$ sodium dodecyl sulfate; $\mathrm{pH} 8.0$ ) in a ratio of $1 \mathrm{~mL}$ of lysate reagent $/ 100 \mathrm{mg}$ of mycelium powder. The total DNA was separated by centrifugation at $11,300 \times g$ for $10 \mathrm{~min}$.

For total RNA extraction, the TRIzol RNA Kit (Life Technologies, Carlsbad, CA, USA) was used, according to the manufacturer's instructions. The concentration and integrity of the extracted total RNA were assessed by the absorbance at $260 \mathrm{~nm}\left(\mathrm{~A}_{260}\right)$ and the $\mathrm{A}_{260} / \mathrm{A}_{280}$ ratio, respectively, and with electrophoresis on $1 \%$ agarose gel.

\section{Construction of gene deletion mutants of $P$. oxalicum}

Gene deletion mutants of $P$. oxalicum were constructed with the homologous recombination method, reported by Zhao et al. [5]. Protoplasts of the parental strain $\triangle$ PoxKu 70 were first prepared. Fresh conidia of $\triangle P$ PoxKu70 were cultured at $28{ }^{\circ} \mathrm{C}$ with shaking at $180 \mathrm{rpm}$ for $8 \mathrm{~h}$ in $200 \mathrm{~mL}$ of CM medium $\left(\mathrm{g} / \mathrm{L}: \mathrm{NaNO}_{3}\right.$ $30, \mathrm{KCl} 2.60, \mathrm{MgSO}_{4} \cdot 7 \mathrm{H}_{2} \mathrm{O} 2.60, \mathrm{KH}_{2} \mathrm{PO}_{4} 7.60$, D-glucose 10.0 , peptone 2.0 , yeast extract 1.0 , and acid-hydrolyzed casein 1.0; $\mathrm{pH}$ 6.5). The grown mycelia were collected, washed three times with $0.6 \mathrm{M} \mathrm{MgSO}_{4} \cdot 7 \mathrm{H}_{2} \mathrm{O}$, and lysed for $2.5 \mathrm{~h}$ in $\mathrm{OM}$ solution $\left(1.2 \mathrm{M} \mathrm{MgSO}_{4} \cdot 7 \mathrm{H}_{2} \mathrm{O}, 10 \mathrm{mM}\right.$ $\mathrm{NaH}_{2} \mathrm{PO}_{4}, 6 \mathrm{~g} / \mathrm{L}$ snailase, $4 \mathrm{~g} / \mathrm{L}$ lysozyme, and $6 \mathrm{~g} / \mathrm{L}$ lysing enzymes from T. harzianum [Sigma-Aldrich, St. Louis, MO, USA]; $\mathrm{pH}$ 5.8). After the addition of trapping buffer (0.4 $\mathrm{M}$ sorbitol, $0.1 \mathrm{M}$ Tris- $\mathrm{HCl}$; $\mathrm{pH}$ 7.0), the protoplasts were collected by centrifugation, precipitated, and washed sequentially with $1 \mathrm{M}$ sorbitol and STC solution (1 $\mathrm{M}$ sorbitol and $0.1 \mathrm{M}$ Tris $-\mathrm{HCl}$, and $0.1 \mathrm{M} \mathrm{CaCl}_{2} ; \mathrm{pH}$ 8.0). The protoplasts were finally resuspended in $0.5 \mathrm{~mL}$ of $4 \times$ STC and $1 \times$ PTC (40\% polyethylene glycol 3350 ,

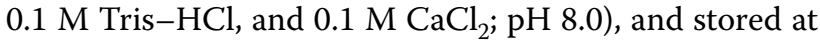
$-80^{\circ} \mathrm{C}$ until analysis.

The knock-out cassette for each candidate gene was constructed with fusion PCR, and included a 1.8-kb DNA fragment encoding the G418-resistance gene and approximately $2 \mathrm{~kb}$ each of the upstream and downstream DNA fragments flanking the target gene. A specific amount of each knock-out cassette was dissolved in $0.1 \mathrm{M}$ spermidine, added to the protoplast suspension, and incubated on ice for $30 \mathrm{~min}$. After incubation, the mixture was transferred to $1 \times$ PTC solution and maintained at room temperature for $25 \mathrm{~min}$. The cultured PTC solution was then added to Petri dishes, mixed with OCM medium containing $1.0 \mathrm{~g} / \mathrm{L}$ casein enzymatic hydrolysate, $1.0 \mathrm{~g} / \mathrm{L}$ yeast extract, $273.6 \mathrm{~g} / \mathrm{L}$ sucrose, and $10.0 \mathrm{~g} / \mathrm{L}$ agar, and incubated at $50{ }^{\circ} \mathrm{C}$ for $30 \mathrm{~min}$. PDA medium containing $500 \mu \mathrm{g} / \mathrm{mL}$ G418 and $250 \mu \mathrm{g} / \mathrm{mL}$ hygromycin B was added to the surface of the OCM medium. After 5 days in culture at $28{ }^{\circ} \mathrm{C}$, the transformants were selected and purified.

\section{Gene complementation}

To construct the complemented strains of the mutants $\triangle P o x C x r A, \triangle P o x C x r B$ and $\triangle P o x N s d D$, complementary cassettes carrying Bleomycin resistance gene were, respectively, constructed, comprised of the upstream sequence of the integrative locus for the complementary cassette, the coding sequence of the complementary gene, the 
Bleomycin resistance gene and the downstream sequence of the integrative locus for the complementary cassette.

For constructing the complementary PoxCxrA cassette, an approximately $5.2 \mathrm{~kb}$ DNA including the upstream sequence of the gene PoxCxrA containing the promoter region, the coding sequence of PoxCxrA and $0.4 \mathrm{~kb}$ of putative terminator of PoxCxrA was obtained via PCR using the specific primer pair CxrA-L-F/CxrA-R. The Bleomycin resistance gene was amplified from the plasmid pZTBle stored in our laboratory using primer pair Ble-F/ Ble-R. An approximately $2.5 \mathrm{~kb}$ DNA containing the 3 '-downstream sequence of $\operatorname{Pox} C x r A$ was amplified from genome of $P$. oxalicum HP7-1 using the primer pair CxrAR-F/CxrA-R-R. The three PCR fragments were ligated using fusion PCR, and then the complementary PoxCxrA cassette was amplified using the nest primer pair C-CxrAF/C-CxrA-R (Additional file 7: Table S4). The resultant cassette was introduced into the $\triangle P o x C x r A$ mutant to generate the complementary strain $C \triangle P o x C x r A$.

The complementary strains $\mathrm{C} \triangle \operatorname{PoxC} \mathrm{Cr} B$ and $C \triangle P o x N s d D$ were constructed similarly as described above. Four DNA fragments, including the left-flanking and right-flanking sequences of an aspartic protease gene PoxPepA (POX05007) in the genome of P. oxalicum HP71 , Bleomycin resistance gene and the complementary gene, were amplified using primer pairs PepA-L-F/PepA-L-R, PepA-R-F/PepA-R-R, Ble-F/Ble-R and CxrB-F/CxrB-F (or NsdD-F/NsdD-R), respectively (Additional file 7: Table S4). The four PCR products were ligated using $p E A S Y^{\circledR}{ }_{-}$ Uni Seamless Cloning and Assembly Kit (TransGen Biotech, Beijing, China). Complementary cassettes of Pox$C x r B$ and PoxNsdD were then generated by PCR using primer pair C-PepA-F/C-PepA-R (Additional file 7: Table S4), and, respectively, integrated into the locus of PoxPepA in genome of the corresponding deletion mutants.

\section{RNA sequencing}

RNA was sequenced with the method described by Zhao et al. [5]. A cDNA library of each sample was constructed for RNA-seq and then assessed with an Agilent 2100 Bioanalyzer (Agilent Technologies, Santa Clara, CA, USA) and the ABI StepOnePlus Real-Time PCR System (Applied Biosystems, Foster City, CA, USA). The Illumina HiSeq 2000 system was used to sequence the cDNA libraries. The raw reads generated were filtered, and the following reads were removed: those including the adapter sequence, those with a high content $(>10 \%)$ of unknown bases, and low-quality reads in which the percentage of low-quality bases $(Q \leq 10)$ was $>50 \%$. The clean reads thus generated were then subjected to quality control (QC) through drawing the base compositions and quality distributions. After QC, the clean reads were mapped onto the genome of $P$. oxalicum wild-type strain
HP7-1 to search for gene homologies and their functional annotations, using the software BWA v0.7.10-r789 [22] and Bowtie2 v2.1.0 [23]. The RSEM v1.2.12 software [24] was used to analyze the gene expression levels (fragments per kilobase of exon per million mapped reads, FPKM). The differentially expressed genes were screened with the NOISeq tool [25], with $\mid \log _{2}$ fold change $\mid>0.8$ and probability $\geq 0.8$ as thresholds. The reliability of RNA-Seq was assessed in three biological replicates of each sample with Pearson's correlation coefficient. These datasets (FPKM) were subjected to a hierarchical cluster analysis using the software Heml 1.0 [33] to determine the groups of genes with similar expression patterns for a different group of regulons. BLAST v2.2.26 (http://blast.ncbi.nlm. nih.gov/Blast.cgi) was used for gene homology and function annotation. The differently expressed genes detected by comparative assays were functionally analyzed based on Kyoto encyclopedia of genes and genomes (KEGG) annotation to Genome of P. oxalicum HP7-1 [5].

\section{Southern hybridization analysis}

The mutants were analyzed with Southern hybridization, as previously described [5]. The genomic DNA of each gene deletion mutant was digested with Apa1 for PoxCxrA, BamH1 for PoxCxrB, and EcoR1 for PoxNsdD (TakaRa Bio Inc., Dalian, China). The enzyme-digested DNA fragments were separated on $0.8 \%$ agarose gel and transferred to Hybond- $\mathrm{N}^{+}$nylon membrane (GE Healthcare Limited, Buckinghamshire, UK). The probes amplified with the indicated primers (Additional file 7: Table S4), using the genomic DNA of HP7-1 as the template, were labeled and detected with the DIG-High Prime DNA Labeling and Detection Starter Kit (Life Technologies, Carlsbad, CA, USA).

\section{Real-time RT-qPCR}

RT-qPCR was used to compare the expression levels of the cellulase and xylanase genes in the deletion mutants and the parental strain $\triangle P o x K u 70$ based on a previously described method [5]. The PrimeScript RT Reagent Kit (TakaRa Bio Inc.) was used to synthesize the first-stand cDNA. The final RT-qPCR mixture $(20 \mu \mathrm{L})$ contained $0.8 \mu \mathrm{L}$ of $10 \mu \mathrm{M}$ primers (Additional file 7: Table S4), $0.2 \mu \mathrm{L}$ of first-stand cDNA as the template, and $10 \mu \mathrm{L}$ of SYBR Premix Ex Taq II (TakaRa Bio Inc.). All the reactions were run for 40 cycles of $95{ }^{\circ} \mathrm{C}$ for $3 \mathrm{~s}$ and $60{ }^{\circ} \mathrm{C}$ for $30 \mathrm{~s}$. The fluorescent signal was detected at the end of each extension step at $80{ }^{\circ} \mathrm{C}$. The relative expression of the target genes was calculated using the actin gene (POX09428) as the control and normalizing all expression to the parental strain $\triangle P o x K u 70$. All RT-qPCRs were repeated independently at least three times. 
Expression of DNA-binding-domain-encoding sequences of PoxCxrA, PoxCxrB, and PoxNsdD, and purification of the recombinant polypeptides

The DNA-binding-domain-encoding sequences of Pox$C x r A$, PoxCxrB, and PoxNsdD were expressed in E. coli Rossetta (Transgen Biotech, Beijing, China). The DNA fragments encoding the DNA-binding domains of PoxCxrA (amino acids 17-150), PoxCxrB (amino acids 181-330), and PoxNsdD (amino acids 335-494) were amplified with PCR using the cDNA of P. oxalicum HP7-1 as the template, with primer pairs PoxCxrA $\mathrm{A}_{17-150}-\mathrm{F} / \mathrm{Pox}-$

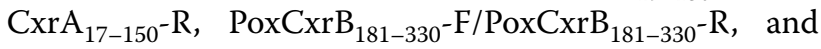
PoxNsdD $335-494-\mathrm{F} /$ PoxNsdD $335-494-\mathrm{R}$, respectively (Additional file 7: Table S4). The PCR products were digested with EcoR1 and/or Not1, and BamH1, and then inserted into the expression vector $\mathrm{pET}-32 \mathrm{a}(+)$ digested with the corresponding restriction endonucleases. The fusion plasmids were introduced into competent E. coli Rossetta cells with chemical transformation. After PCR confirmation, the recombinant strains were cultured in LuriaBertani medium for $8 \mathrm{~h}$, and then induced with $0.5 \mathrm{mM}$ isopropyl- $\beta$-D-thiogalactopyranoside at $25{ }^{\circ} \mathrm{C}$ to produce recombinant proteins fused with TRX, His, and $\mathrm{S}$ tags. The strain containing the empty vector $\mathrm{pET}-32 \mathrm{a}(+)$ was cultured as the control.

After the cells were disrupted with ultrasonication, the fusion proteins were purified with affinity chromatography on TALON Metal Affinity Resin (Clontech, Palo Alto, CA, USA), according to the instructions of the manufacturer. The protein concentrations were measured with the Bradford Protein Assay Kit (Tiangen, Beijing, China), according to the manual.

\section{EMSA}

DNA fragments (100-500 bp upstream from the ATG start codons of PoxCxrA, PoxCxrB, PoxNsdD, POX01166/ Cel5B, POX05587/Cel7A-2, POX06835/Bgl3A, and $P O X 06783 / X y n 11 A)$ were generated with PCR and the corresponding primers (Additional file 7: Table S4). Each reverse primer was labeled with FAM at its $3^{\prime}$-terminus. After purification, $40 \mathrm{ng}$ of each FAM-labeled PCR product was mixed with various amounts $(0-4.8 \mu \mathrm{g})$ of DNAbinding-domain polypeptide of the regulator proteins in a binding buffer $(0.1 \mathrm{mg} / \mathrm{mL}$ BSA, $20 \mathrm{mM}$ Tris- $\mathrm{HCl}[\mathrm{pH}$ 8.0], $5 \%$ glycerol, $50 \mathrm{mM} \mathrm{KCl,} 1 \mathrm{mM}$ dithiothreitol). For the competitive binding experiments, a known amount of binding protein was mixed with various amounts of probe under the conditions described above. The protein-DNA complexes were separated with $4 \%$ polyacrylamide-Trisacetic acid-EDTA (TAE) gel electrophoresis, and visualized with the Bio-Rad ChemiDoc ${ }^{\mathrm{TM}}$ MP Imaging System (Bio-Rad Laboratories, Inc. Hercules, CA, USA) at an excitation wavelength of 489-506 nm. Each protein-DNA complex was retarded relative to the free DNA. BSA alone or TRX-His-S purified from the total protein extracted from $E$. coli cells containing the empty vector pET-32a $(+)$ was used as the negative control.

\section{Enzyme activities and protein concentrations}

The cellulase and xylanase activities and protein concentrations were measured as described previously [5]. Briefly, FPase activity was measured with Whatman No. 1 filter paper $\left(50 \mathrm{mg}, 1.0 \times 6.0 \mathrm{~cm}^{2}\right)(\mathrm{GE}$ Healthcare Company) as the substrate in $1.0 \mathrm{~mL}$ of $100 \mathrm{mM}$ citrate buffer (pH 5.0) and $0.5 \mathrm{~mL}$ of suitably diluted crude cellulase for $1 \mathrm{~h}$ at $50{ }^{\circ} \mathrm{C}$. CMCase activity was tested by incubating 1.0\% CMC-Na (Sigma-Aldrich, Darmstadt, Germany) solution in citrate buffer $(100 \mathrm{mM}, \mathrm{pH} 5.0)$ containing a specific amount of crude cellulase for $30 \mathrm{~min}$ at $50{ }^{\circ} \mathrm{C}$. Xylanase activity was measured under similar conditions, except that the substrate was replaced with $1.0 \%$ xylan from beechwood (Megazyme International Ireland, Wicklow, Ireland), and the incubation time was $10 \mathrm{~min}$. After two volumes of 3,5-dinitrosalicyclic acid were added, the reducing sugars generated were measured at $540 \mathrm{~nm}$. One unit of enzymatic activity (U) was defined as the amount of enzyme required to produce $1 \mu \mathrm{mol}$ of reducing sugar per min from the reaction substrates. Triplicate independent experiments were performed for each sample.

To measure the pNPCase and pNPGase activities, $p$-nitrophenyl- $\beta$-D-cellobioside (pNPC) and $p$-nitrophenyl $\beta$-D-glucopyranoside (pNPG) (Sigma-Aldrich) were used as the substrates, respectively, liberating $p$-nitrophenol at $50{ }^{\circ} \mathrm{C}$ and $\mathrm{pH} 5.0$ after incubation for $15 \mathrm{~min}$ in $140 \mu \mathrm{L}$ reaction systems $(116 \mu \mathrm{L}$ of $100 \mathrm{mM}$ citrate buffer, $14 \mu \mathrm{L}$ of $25 \mathrm{mM}$ substrate, and $10 \mu \mathrm{L}$ of diluted crude enzyme). Sodium carbonate $(0.4 \mathrm{M}, 70 \mu \mathrm{L})$ was used to stop the reactions. The $p$-nitrophenol produced was measured with spectrometry at $410 \mathrm{~nm}$. One unit of enzymatic activity (U) was defined as the amount of enzyme that produced $1 \mu \mathrm{mol}$ of $p$-nitrophenol per min from the appropriate substrate. Each sample was analyzed independently at least three times.

The protein concentrations were measured with a Pierce $^{\mathrm{TM}}$ detergent compatible Bradford assay kit (Pierce Biotechnology, Rockford, IL, USA), according to the manufacturer's instructions.

\section{Phylogenetic analysis}

The amino acid sequences of PoxCxrA, PoxCxrB, and PoxNsdD homologues were downloaded from the NCBI BlastP website (https://blast.ncbi.nlm.nih.gov/Blast.cgi). A phylogenetic tree was constructed using the MEGA version 7.0 software [34] with the neighbor-joining method and a Poisson correction model. In this process, 1000 replicates were used to calculate the bootstrap values. 


\section{Statistical analysis}

Student's $t$ test (two-tailed) in Microsoft Excel (Office 2016) (Microsoft, Redmond, WA, USA) was used for the statistical analysis of the data.

\section{Accession numbers}

All transcriptomic data are available from the Sequence Read Archive database (Accession Number SRA505232). DNA sequences are available from the GenBank database (Accession Numbers KY368171-KY368173, KY922971, and KY860734-KY860740).

\section{Additional files}

Additional file 1: Figure S1. Cellulase activity of P. oxalicum HP7-1 in the presence of glucose $(\mathrm{Glu})$, wheat bran (WB), or wheat bran and Avicel (WA). Data are the means of three biological replicates.

Additional file 2: Table S1. Summary of RNA-sequencing reads obtained for P. oxalicum strain HP7-1 and its derived mutants.

Additional file 3: Figure S2. Pearson's correlation analysis of the transcriptomes of P. oxalicum HP7-1 in the presence of glucose (Glu), wheat bran (WB), or wheat bran and Avicel (WA) as the carbon source. RNA for sequencing was extracted from cells sampled $72 \mathrm{~h}$ after inoculation.

Additional file 4: Table S2. List of 108 genes differentially coexpressed on all carbon sources tested, including glucose (Glu), wheat bran (WB), and wheat bran and Avicel (WA).

Additional file 5: Table S3. Forty candidate transcription factors regulating the expression of cellulase and xylanase genes in P. oxalicum detected with a transcriptomic profiling analysis.

Additional file 6: Figure S3. Confirmation analysis of the deletion mutants of 31 candidate genes derived from the parental strain $\triangle P$ PoxKu 70 . (A-AE) PCR analysis of: (A) $\triangle P O X 00864$; (B) $\triangle P$ OXCIrC; (C) $\triangle P O X 01167 / P O X-$ CXrA; (D) $\triangle P O X 01183 ;$ (E) $\triangle P O X 01184 ;$ (F) $\triangle P O X 02261 ;$ (G) $\triangle P O X 02682 ;$ (H) $\triangle P O X 02944 ;(\mathrm{l}), \triangle P O X 03888 ;(J) \triangle P O X 03910 ;(\mathrm{K}) \triangle P O X 04193 ;$ (L) $\triangle P O X 04420 /$ PoXCXrB; (M) $\triangle P O X 04590 ;(\mathrm{N}) \triangle P O X 04676 ;$ (O) $\triangle P O X 04772 ;$ (P) $\triangle P O X 04860$; (Q) $\triangle P O X 05374 ;$ (R) $\triangle P O X 05436 ;$ (S), $\triangle P O X 05726 ;$ (T) $\triangle P O X 06377 ;(\mathrm{U})$ $\triangle P O X 06396 ;(\mathrm{V}) \triangle P O X 06425 ;$ (W) $\triangle P O X B r I A ;(X) \triangle P O X 06759 ;(Y) \triangle P O X F 1 b D$; (Z) $\triangle P O X 07934$; (AA) $\triangle P O X 08415 / P 0 X N s d D$; (AB) $\triangle P O X 08702$; (AC) $\triangle P O X 08910$; (AD) $\triangle P O X 09356$; and (AE) $\triangle P O X 09460 . \mathrm{M}, 1-\mathrm{kb}$ DNA marker: lanes $1-3$, three transformants constructed for each candidate gene; lane 4, $\triangle$ POXKu70; lane 5, dd $\mathrm{H}_{2} \mathrm{O}$. (AF-AH) Southern hybridization analysis: (AF) $\triangle P O X 01167 / P 0 \times C X r A ; M, 1-k b$ DNA marker; lane 1, $\triangle P$ OxKu70; lane 2, $\triangle P O X 01167 / P O X C X \times A-6 ;$ lane 3, $\triangle P O X 01167 / P O X C X r A-9 ;$ lane 4, $\triangle P O X 01167 /$ PoXCXYA-11. (AG) $\triangle P O X 04420 ; \mathrm{M}, 1-\mathrm{kb}$ DNA marker; 1, $\triangle P$ OXKu70; 2 , $\triangle P O X 04420 / P O X C X r B-2 ; 3, \triangle P O X 04420 / P O X C X r B-6 ; 4, \triangle P O X 04420 / P 0 X-$ CXrB-8. (AH) $\triangle P O X 08415 ; \mathrm{M}, 1$-kb DNA marker; lane 1, $\triangle P$ OXKu 70; lane 2, $\triangle P O X 08415 / P o x N s d D-1 ;$ lane 3, $\triangle P O X 08415 / P o x N s d D-5$; lane 4, $\triangle P O X 08415$ / PoxNsdD-9.

Additional file 7: Table S4. Primers used in this study.

Additional file 8: Figure S4. Unrooted phylogenetic tree of PoxCxrA, PoxCxrB, and PoxNsdD and their putative homologues. The dendrogram was constructed with the MEGA 7 software using the neighbor-joining method and a Poisson model. Bootstrap values shown at the nodes were derived with 1000 replicates, and the branch lengths, which correspond to the divergence of the sequences, are indicated by the scale bar.

Additional file 9: Figure S5. Confirmation analysis of the complementary strains. Targeted complementary genes including PoxCXrA, PoxCXrB and PoxNsdD were amplified using primer pairs CxrA-CDS-F/CXrA-CDSR, CxrB-CDS-F/CxrB-CDS-R and NsdD-CDS-F/NsdD-CDS-R. Bleomycin resistance gene was amplified using primer pair Ble-F/Ble-R. M, 1-kb
DNA marker; lane 1, complementary strain; lane 2, $\triangle$ PoxKu70; lane 3, corresponding deletion mutant strain.

Additional file 10: Figure S6. Electrophoretic mobility shift assay showing the interaction between the DNA-binding domains of the regulators and the promoter sequences of cellulase and xylanase genes. The experiments were performed without competitive probes.

Additional file 11: Figure S7. Electrophoretic mobility shift assay showing the interaction between the DNA-binding domains of the regulators and the promoter sequences of the newly identified regulatory genes. The experiments were performed without competitive probes.

\section{Abbreviations}

CWDEs: plant-cell-wall-degrading enzymes; EMSA: electrophoretic mobility shift assay; Glu: glucose; HMG: high-mobility group; PDA: potato-dextrose agar; RT-qPCR: quantitative reverse transcription-PCR; TF: transcription factor; TRX: thioredoxin; WA: wheat bran plus Avicel; WB: wheat bran; CMCase: carboxymethylcellulase; FPase: filter-paper cellulase; pNPCase: $p$-nitrophenyl$\beta$-cellobiosidase; pNPGase: $p$-nitrophenyl- $\beta$-glucopyranosidase; EG: endoglucanase; CBH: cellobiohydrolase; BGL: $\beta$-glucosidase; MMM: modified minimal medium; FPKM: fragments per kilobase of exon per million mapped fragments.

\section{Authors' contributions}

JXF designed and supervised the research, was involved in the data analysis and the preparation of the manuscript. SZ co-supervised all the research, wrote and revised the manuscript, and was involved in the analysis of all experimental data. YSY did the experiments of comparative transcriptome, constructed deletion mutants of 11 candidate genes and subsequently tested enzyme activities, discovered the key transcription factor PoxCxrA and performed PoxCxrA-related experiments e.g. RT-qPCR, cellulase activity measurement, complementation and EMSA of POxCXrA. LSL constructed deletion mutants of 5 candidate genes and subsequently tested enzyme activities, discovered the novel transcription factor PoxCxrB and performed PoxCxrB-related experiments. QPH constructed deletion mutants of 8 candidate genes and subsequently tested enzyme activities, discovered the novel transcription factor PoxNsdD and performed PoxNsdD-related experiments. YRX constructed deletion mutants of 7 candidate genes and subsequently tested enzyme activities. LW was involved in the design of mutant construction and analysis of some experimental data. CXL was involved in the bioinformatic analysis of the transcriptomes. All authors read and approved the final manuscript.

\section{Acknowledgements}

We would like to thank Jin-Qun Huang, Shang-Bo Xie, and Lin Yang at BGIShenzhen, China, for their assistance with the RNA sequencing and transcriptomic data analysis.

\section{Competing interests}

The authors declare that they have no competing interests.

\section{Availability of supporting data}

All transcriptomic data are available from the Sequence Read Archive database (accession number SRA505232). DNA sequences are available from the GenBank database (accession numbers KY368171-KY368173, KY922971, and KY860734-KY860740).

\section{Consent for publication}

All authors consent to the publication of this manuscript.

\section{Ethical approval and consent to participate}

Not applicable.

\section{Funding}

This work was supported financially by the Guangxi BaGui Scholars Program Foundation (Grant No. 2011 A001) to JXF, grants from the National Natural Science Foundation of China (http://www.nsfc.gov.cn/) (Grant Nos 31260017 and 31660305) to JXF and SZ, and the 'One Hundred Person' Project of Guangxi to SZ. 


\section{Publisher's Note}

Springer Nature remains neutral with regard to jurisdictional claims in published maps and institutional affiliations.

Received: 14 April 2017 Accepted: 10 November 2017

Published online: 22 November 2017

\section{References}

1. Tiwari R, Nain L, Labrou NE, Shukla P. Bioprospecting of functional cellulases from metagenome for second generation biofuel production: a review. Crit Rev Microbiol. 2017;13:1-14.

2. Gusakov AV. Alternatives to Trichoderma reesei in biofuel production. Trends Biotechnol. 2011;29:419-25.

3. Zhang Z, Liu JL, Lan JY, Duan CJ, Ma QS, Feng JX. Predominance of Trichoderma and Penicillium in cellulolytic aerobic filamentous fungi from subtropical and tropical forests in China, and their use in finding highly efficient beta-glucosidase. Biotechnol Biofuels. 2014;7:107.

4. Jing $L$, Zhao S, Xue JL, Zhang Z, Yang Q, Xian L, Feng JX. Isolation and characterization of a novel Penicillium oxalicum strain Z1-3 with enhanced cellobiohydrolase production using cellulase-hydrolyzed sugarcane bagasse as carbon source. Ind Crop Prod. 2015;77:666-75.

5. Zhao S, Yan YS, He QP, Yang L, Yin X, Li CX, Mao LC, Liao LS, Huang JQ, Xie SB, Nong QD, Zhang Z, Jing L, Xiong YR, Duan CJ, Liu JL, Feng JX. Comparative genomic, transcriptomic and secretomic profiling of Penicillium oxalicum HP7-1 and its cellulase and xylanase hyper-producing mutant EU2106, and identification of two novel regulatory genes of cellulase and xylanase gene expression. Biotechnol Biofuels. 2016;9:203.

6. Amore A, Giacobbe S, Faraco V. Regulation of cellulase and hemicellulase gene expression in fungi. Curr Genom. 2013;14:230-49.

7. Tani S, Kawaguchi T, Kobayashi T. Complex regulation of hydrolytic enzyme genes for cellulosic biomass degradation in filamentous fungi. Appl Microbiol Biotechnol. 2014;98:4829-37.

8. van Peij NN, Gielkens MM, de Vries RP, Visser J, de Graaff LH. The transcriptional activator $X I n R$ regulates both xylanolytic and endoglucanase gene expression in Aspergillus niger. Appl Environ Microbiol. 1998;64:3615-9.

9. Noguchi Y, Sano M, Kanamaru K, Ko T, Takeuchi M, Kato M, Kobayashi T. Genes regulated by AoXInR, the xylanolytic and cellulolytic transcriptional regulator, in Aspergillus oryzae. Appl Microbiol Biotechnol. 2009:85:141-54.

10. Coradetti ST, Craig JP, Xiong Y, Shock T, Tian CG, Glass NL. Conserved and essential transcription factors for cellulase gene expression in ascomycete fungi. Proc Natl Acad Sci USA. 2012;109:7397-402.

11. Coradetti ST, Xiong Y, Glass NL. Analysis of a conserved cellulase transcriptional regulator reveals inducer-independent production of cellulolytic enzymes in Neurospora crassa. Microbiologyopen. 2013;2:595-609.

12. Xiong Y, Sun JP, Glass NL. VIB1, a link between glucose signaling and carbon catabolite repression, is essential for plant cell wall degradation by Neurospora crassa. PLoS Genet. 2014;10:e1004500.

13. Katz ME, Gray KA, Cheetham BF. The Aspergillus nidulans xprG (phoG) gene encodes a putative transcriptional activator involved in the response to nutrient limitation. Fungal Genet Biol. 2006;43:190-9.

14. Hakkinen M, Valkonen MJ, Westerholm-Parvinen A, Aro N, Arvas M, Vitikainen M, Penttila M, Saloheimo M, Pakula TM. Screening of candidate regulators for cellulase and hemicellulase production in Trichoderma reesei and identification of a factor essential for cellulase production. Biotechnol Biofuels. 2014;7:14

15. Huberman LB, Liu J, Qin LN, Glass NL. Regulation of the lignocellulolytic response in filamentous fungi. Fungal Biol Rev. 2016;30:101-11.

16. Antonieto AC, dos Santos Castro L, Silva-Rocha R, Persinoti GF, Silva RN. Defining the genome-wide role of CRE1 during carbon catabolite repression in Trichoderma reesei using RNA-Seq analysis. Fungal Genet Biol. 2014;73:93-103.

17. Li ZH, Yao GS, Wu RM, Gao LW, Kan QB, Liu M, Yang P, Liu GD, Qin YQ, Song $X$, Zhong YH, Fang X, Qu YB. Synergistic and dose-controlled regulation of cellulase gene expression in Penicillium oxalicum. PLoS Genet. 2015;11:e1005509.
18. Ries LNA, Beattie SR, Espeso EA, Cramer RA, Goldman GH. Diverse regulation of the CreA carbon catabolite repressor in Aspergillus nidulans. Genetics. 2016:203:335-52.

19. Yao G, Li Z, Gao L, Wu R, Kan Q, Liu G, Qu Y. Redesigning the regulatory pathway to enhance cellulase production in Penicillium oxalicum. Biotechnol Biofuels. 2015;8:71.

20. Gao L, Li Z, Xia C, Qu Y, Liu M, Yang P, Yu L, Song X. Combining manipulation of transcription factors and overexpression of the target genes to enhance lignocellulolytic enzyme production in Penicillium oxalicum. Biotechnol Biofuels. 2017;10:100.

21. Sun X, Liu Z, Qu Y, Li X. The effects of wheat bran composition on the production of biomass-hydrolyzing enzymes by Penicillium decumbens. Appl Biochem Biotechnol. 2008;146:119-28.

22. Li H, Durbin R. Fast and accurate short read alignment with BurrowsWheeler transform. Bioinformatics. 2009;25:1754-60.

23. Langmead B, Salzberg SL. Fast gapped-read alignment with Bowtie 2. Nat Methods. 2012;9:357-9

24. Li B, Dewey CN. RSEM: accurate transcript quantification from RNA-Seq data with or without a reference genome. BMC Bioinform. 2011;12:323.

25. Huber W, Carey VJ, Gentleman R, Anders S, Carlson M, Carvalho BS, Bravo HC, Davis S, Gatto L, Girke T, Gottardo R, Hahne F, Hansen KD, Irizarry RA, Lawrence M, Love MI, MacDonald J, Obenchain V, Oleś AK, Pagès H, Reyes A, Shannon P, Smyth GK, Tenenbaum D, Waldron L, Morgan M. Orchestrating high-throughput genomic analysis with bioconductor. Nat Methods. 2015;12:115-21.

26. Li J, Liu G, Chen M, Li Z, Qin Y, Qu Y. Cellodextrin transporters play important roles in cellulase induction in the cellulolytic fungus Penicillium oxalicum. Appl Microbiol Biotechnol. 2013;97:10479-88.

27. Qin Y, Bao L, Gao M, Chen M, Lei Y, Liu G, Qu Y. Penicillium decumbens BrlA extensively regulates secondary metabolism and functionally associates with the expression of cellulase genes. Appl Microbiol Biotechnol. 2013;97:10453-67.

28. Kunitake E, Hagiwara D, Miyamoto K, Kanamaru K, Kimura M, Kobayashi T. Regulation of genes encoding cellulolytic enzymes by Pal-PacC signaling in Aspergillus nidulans. Appl Microbiol Biotechnol. 2016;100:3621-35.

29. Lee MK, Kwon NJ, Lee IS, Jung S, Kim SC, Yu JH. Negative regulation and developmental competence in Aspergillus. Sci Rep. 2016;6:28874.

30. Han KH, Han KY, Yu JH, Chae KS, Jahng KY, Han DM. The nsdD gene encodes a putative GATA-type transcription factor necessary for sexual development of Aspergillus nidulans. Mol. Microbiol. 2001:41:299-309.

31. Lee MK, Kwon NJ, Choi JM, Lee IS, Jung S, Yu JH. NsdD is a key repressor of asexual development in Aspergillus nidulans. Genetics. 2014;197:159-73.

32. Chen M, Qin Y, Cao Q, Liu G, Li J, Li Z, Zhao J, Qu Y. Promotion of extracellular lignocellulolytic enzymes production by restraining the intracellular beta-glucosidase in Penicillium decumbens. Bioresour Technol. 2013;137:33-40.

33. Deng WK, Wang YB, Liu ZX, Cheng H, Xue Y. Heml: a toolkit for illustrating heatmaps. PLoS ONE. 2014;9:e111988.

34. Kumar S, Stecher G, Tamura K. MEGA7: molecular evolutionary genetics analysis version 7.0 for bigger datasets. Mol Biol Evol. 2016;33:1870-4.

\section{Submit your next manuscript to BioMed Central and we will help you at every step:}

- We accept pre-submission inquiries

- Our selector tool helps you to find the most relevant journal

- We provide round the clock customer support

- Convenient online submission

- Thorough peer review

- Inclusion in PubMed and all major indexing services

- Maximum visibility for your research

Submit your manuscript at www.biomedcentral com/submit 